\title{
ERROR AND UNCERTAINTY QUANTIFICATION \\ AND SENSITIVITY ANALYSIS \\ IN MECHANICS COMPUTATIONAL MODELS
}

\author{
By \\ Bin Liang \\ Thesis \\ Submitted to the Faculty of the \\ Graduate School of Vanderbilt University \\ In partial fulfillment of the requirements \\ for the degree of \\ MASTER OF SCIENCE \\ in \\ Civil Engineering \\ August, 2010 \\ Nashville Tennessee \\ Approved \\ Professor Sankaran Mahadevan \\ Professor Prodyot K. Basu
}


To my parents and my fiancée 


\section{ACKNOWLEDGEMENT}

This work would not have been possible without the guidance and support of many people. I thank all of my Thesis Committee members for their advice and suggestions. Especially my deepest gratitude is to my thesis supervisor and mentor, Dr. Sankaran Mahadevan. I am very grateful to your guidance, inspiration, patience throughout my stay at Vanderbilt. I would like to thank Dr. Prodyot K. Basu for his advice, suggestions and direction on my graduate study.

I truly appreciate the friendship and technical support I received from You Ling and Sankaranman Shankar throughout my graduate study. I am also thankful for the help and valuable discussions from our research group members, Dr. Mark McDonald, Dr. Chris Shantz, Venkata Sura, Dr. Vadiraj Hombal and Dr. Sirisha Rangavajhala. Also thanks to Dr. Angel Urbina and Dr. Kais Zaman for their inspiration. It is a great pleasure to be in such a creative team.

I would like to extend my thanks to the Air Force Office of Scientific Research

(Project Manager: Dr. Fariba Fahroo), VEXTEC Corporation (Investigators: Dr. Robert Tryon, Dr. Animesh Dey) and Department of Civil and Environmental Engineering at Vanderbilt for their financial support.

Finally I want to extend my most gracious and special thanks to my parents and my fiancée. Without your support and encouragement this could not have been completed. 
TABLE OF CONTENTS

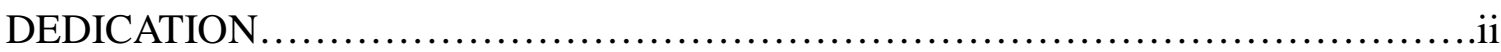

ACKNOWLEDGEMENT.............................................................ii

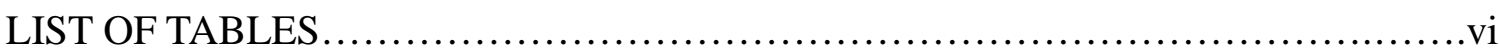

LIST OF FIGURES................................................................

Chapter $\quad$ Page

I. INTRODUCTION

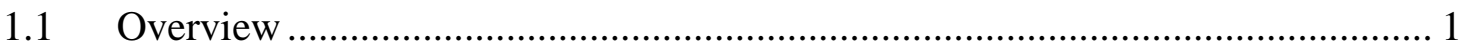

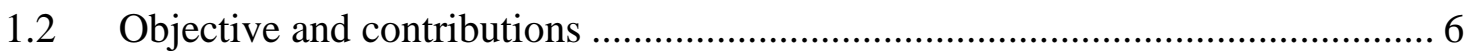

1.3 Organization of the thesis.......................................................................... 7

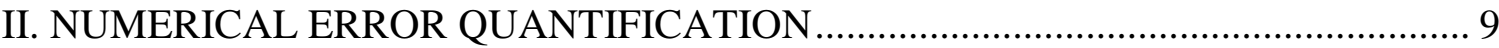

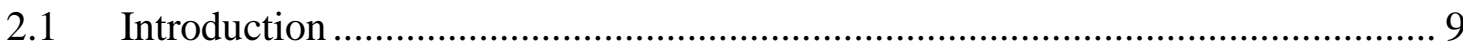

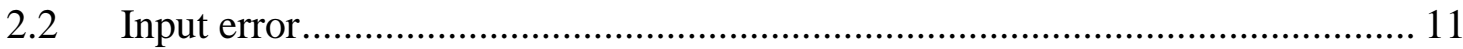

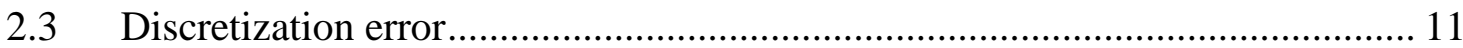

$2.4 \quad$ Surrogate model prediction error ……………….......................................... 14

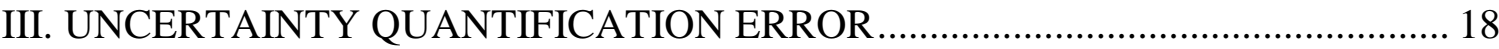

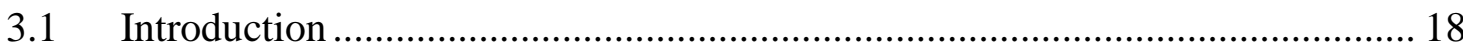

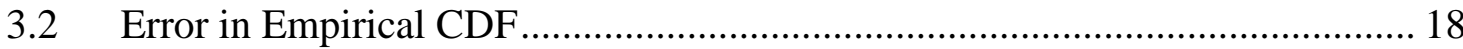

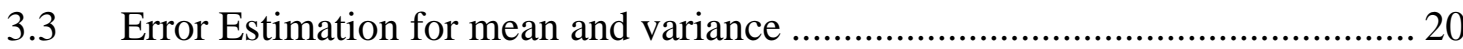

3.4 Propagation of UQ error............................................................................ 24

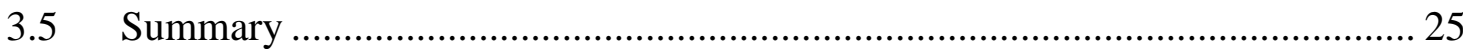

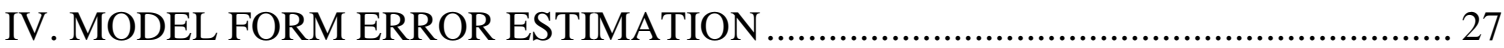

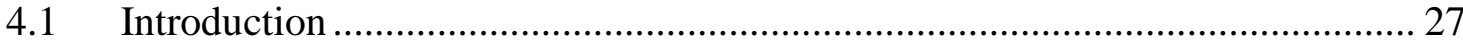

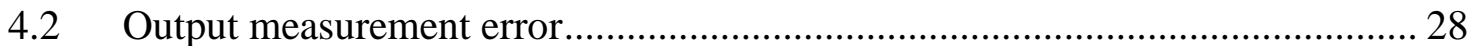

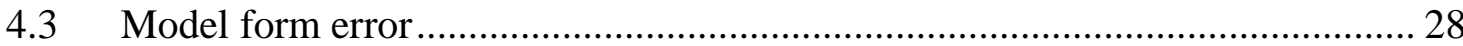

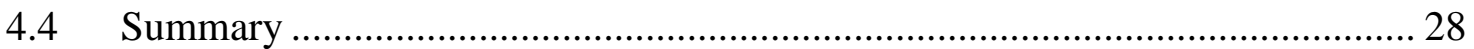

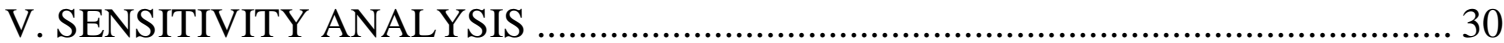




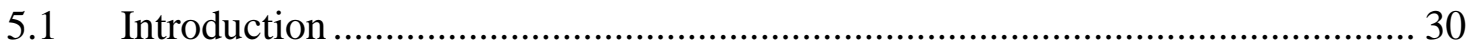

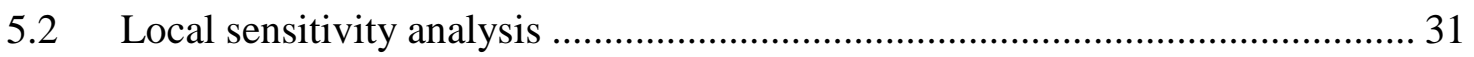

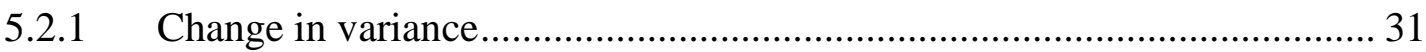

5.2.2 Kullback-Leibler divergence ...................................................................... 31

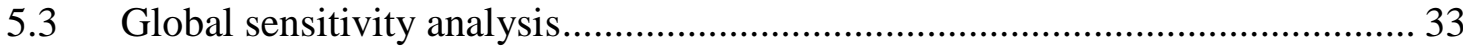

5.3.1 Main effect and total effect measures ...................................................... 33

5.4 An intuitive understanding of the sensitivity measures .................................... 34

5.5 Deterministic and stochastic errors in sensitivity analysis ................................. 36

5.5.1 Example: sensitivity analysis on deterministic error and stochastic error.. 37

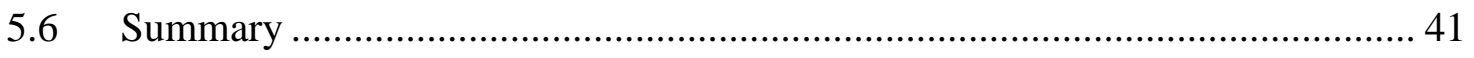

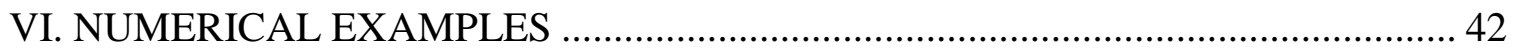

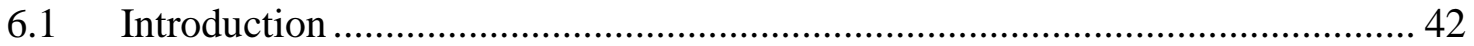

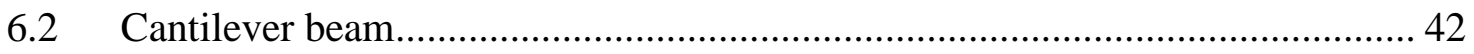

6.3.1. Numerical error estimation ..................................................................... 44

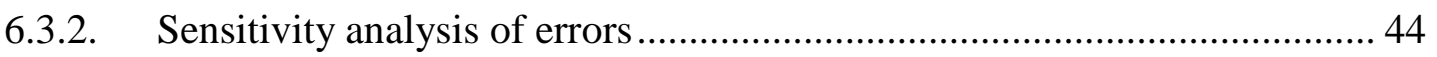

6.3.3. Model form error and UQ error estimation................................................... 48

6.3 Crack growth in an airplane wing spar.......................................................... 50

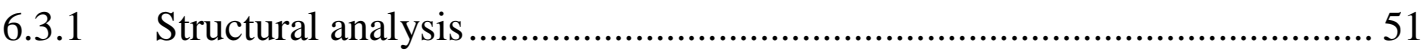

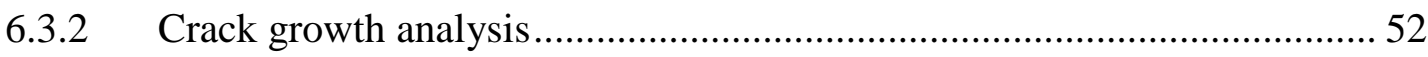

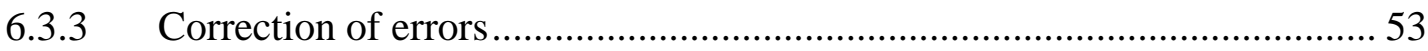

6.3.4 Results and sensitivity analysis................................................................ 53

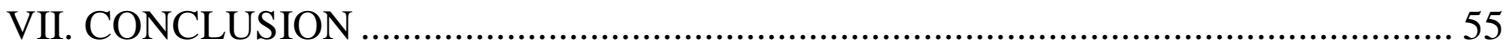

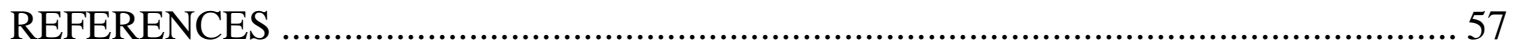




\section{LIST OF TABLES}

Table 1. 15 FEA results of the slender cantilever beam problem .................................. 17

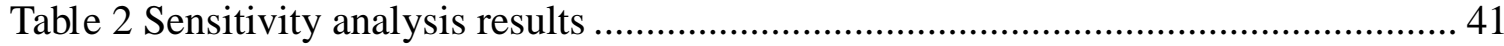

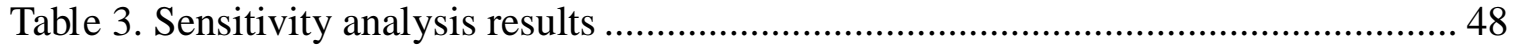

Table 4. Statistics of model form error and sampling errors .......................................... 49

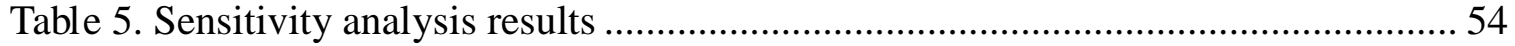




\section{LIST OF FIGURES}

Figure 1. Surrogate model prediction and confidence bounds ........................................ 17

Figure 2. Empirical CDF with 90\% confidence bounds vs. true CDF .............................. 20

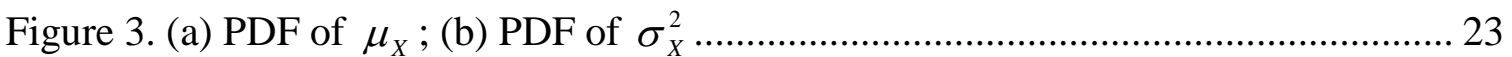

Figure 4. Sampling error quantification.......................................................................... 25

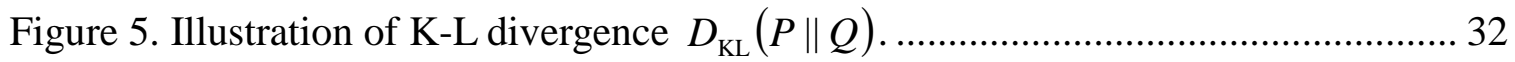

Figure 6. Scatter plots: (a) $Y$ versus $X_{i}$ (b) $Y$ versus $X_{i}$ (c) $E_{X_{\alpha_{i}}}\left(Y \mid X_{i}\right)$ versus $X_{i} \ldots 36$

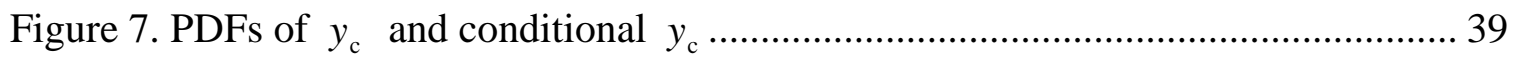

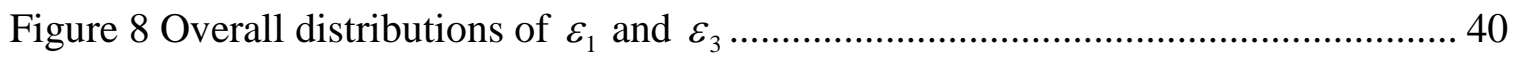

Figure 9. Cantilever beam............................................................................................... 43

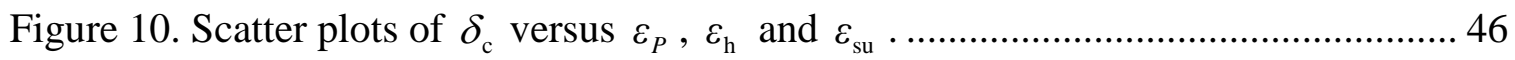

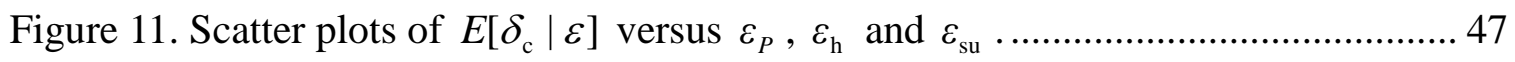

Figure 12. (a): PDFs for model form error (b): PDFs for sampling errors ....................... 49

Figure 13. Component analyses and associated sources of error and uncertainty............ 50

Figure 14. Loading history on the structure ....................................................................... 51 


\section{CHAPTER I}

\section{INTRODUCTION}

\subsection{Overview}

Computational models are widely used by engineers to capture the behavior of physical systems. For large systems, computational models are usually constructed based on sparse experimental data, sometimes even with no full-scale experiments. As a result, errors arise due to lack of data and lack of knowledge about system behavior. Numerical approaches used to solve the model equations also produce errors and uncertainties due to various assumptions and approximations. Natural variability in many physical variables, and data uncertainty due to sparse data and measurement errors, add further uncertainty in the model prediction. Therefore, computational models must be subjected to rigorous model verification and validation $(\mathrm{V} \& \mathrm{~V})$ [1], during which sources of error and uncertainty need to be analyzed and quantified in order to assess the credibility of the model prediction.

Verification and validation are quantitative procedures to check how well the model represents the real world phenomenon being simulated. The accuracy of the solution is assessed with respect to known solutions and experimental data, respectively, 
in verification and validation [2]. An important consequence of $\mathrm{V} \& \mathrm{~V}$ is the identification, quantification and reduction of the errors in the computational model [3].

The error in a computational model prediction consists of two parts: model form error $\left(\varepsilon_{\text {model }}\right)$ and solution approximation error or numerical error $\left(\varepsilon_{\text {num }}\right)[1,4]$. The model form error depends on whether the selected model correctly represents the real phenomenon. For example, engineers might have to choose between a small deformation model and a large deformation model, between a linear elastic model and an elastoplastic model, or between the Euler equation and the Navier-Stokes equation, or between 1-D, 2D and 3-D model. Choosing the correct model could reduce model form error. The solution approximation error arises when numerically solving the model equations, and arises from approximations in the solution procedure. In other words, the model form error is related to the question "Did I choose the correct equation?" which is answered using validation experiments, while the solution approximation error is related to the question "Did I solve the equations correctly?" which is answered through verification studies.

Mathematical theory and methods have been discussed in [4] for quantifying numerical error and model form error in computational mechanics models, but these methods require access to the original PDEs of the system. A simplified approach to error quantification for generic computational models has been developed in [5]. If we denote $y_{\text {pred }}$ and $y_{\text {true }}$ as model prediction and the true response of the physical system, respectively, then we have: 


$$
y_{\text {true }}=y_{\text {pred }}+\varepsilon_{\text {num }}+\varepsilon_{\text {model }}
$$

Rebba and Mahadevan [5] further decomposed the numerical error into various error sources and observed that the numerical error is a nonlinear combination of these components. However, only a simple example was shown with only one error source, namely, finite element discretization error. This research first considers three typical numerical error components and their quantification and combination, including input error, discretization error in FEA, and surrogate model error.

In order to quantify the numerical error, assume $y_{c}$ is the model prediction corrected for error sources, and $y_{\text {pred }}$ is the raw model prediction. Uncertainty propagation analysis is required when calculating $y_{c}$ that includes the uncertainty of error sources. As a result, extra uncertainty is introduced in $y_{c}$, compared to that in $y_{\text {pred }}$. As we will see in Chapter 4, $y_{c}$ is also used to quantify the model form error. Besides $y_{\mathrm{c}}$, to quantify the model form error, observed data $\left(y_{\mathrm{obs}}\right)$ are needed. However, there is a difference between $y_{\mathrm{obs}}$ and $y_{\text {true }}$, which is called output measurement error $\left(\varepsilon_{\mathrm{om}}\right)$. Thus we have

$$
y_{\text {true }}=y_{\text {obs }}+\varepsilon_{\text {om }}
$$

Model form error can be quantified based on Eq. (1.1) and (1.2). Implementation details are discussed in Chapter 4.

One focus of this research is how to obtain a model prediction $y_{c}$ corrected for numerical error. This is a crucial part for model $V \& V$, where the corrected model 
prediction $y_{c}$ plays an important role in both numerical error quantification and model form error quantification. Among all errors, some errors are stochastic, such as input error and surrogate model error, and some errors are deterministic, such as discretization error in FEA. In previous work, model form error is first explicitly expressed in terms of all error sources, and then it is quantified by sampling each of the error terms[5]. This approach has two significant drawbacks: first, in some cases it is not easy to find an analytical expression for model form error in terms of all error sources; second, the discretization error is treated as a random variable, which is incorrect, although we agree on the fact that in non-deterministic analysis correcting for this error would change the uncertainty of model prediction[5, 6, 7]. In this research, a simple but efficient approach is developed to obtain $y_{\mathrm{c}}$. The basic idea is to quantify and correct for each error where it arises. The advantage of this method is that it is easy to implement even if the model involves multiple disciplinary analyses. Stochastic error is corrected for by adding its samples to the original result. Deterministic error is corrected for by directly adding it to the corresponding result. For example, to correct for the discretization error, every time a particular FEA result is obtained, a corresponding discretization error is calculated and is added to the original result. And the new FEA result will be used for further calculation to obtain $y_{c}$.

In addition to the errors mentioned above, another error arises due to the MonteCarlo sampling method used in the error quantification procedure. This error is referred to as uncertainty quantification error (UQ error). For example, when estimating the CDF 
for a random variable from sparse data, UQ error arises as the error in the CDF value. Methods for quantifying this error are available in $[8,9]$. Then if more samples are generated by inverse CDF method using such CDF estimated from sparse data, the UQ error is propagated as sampling error to the newly generated samples. An approach is developed in Section 3.4 to quantify this sampling error. Basically, the proposed approach is a generic re-sampling method from sparse data based on inverse CDF method, considering the error that arises in this procedure. In this research, this method is only applied for model form error quantification (see Chapter 4 for details), just for the sake of demonstration. However, as a generic method, it can be applied anywhere sampling from sparse data is needed, e.g., sampling from sparse input data. Compared to the other resampling methods, e.g. bootstrapping [10] and Johnson distribution [11], this method provides a convenient way to include the uncertainty due to sparseness of data into the new samples.

After a probabilistic framework to manage all sources of uncertainty and error is established, sensitivity analysis is performed to assess the contribution of each source of uncertainty and error to the overall uncertainty in the corrected model prediction. The sensitivity analysis result can be used to effectively make improvements according to the importance ranking of the errors so as to trade off between accuracy and computational effort. 


\subsection{Objective and contributions}

The objective of this research is to develop a methodology that provides information regarding the relative contribution of various sources of error and uncertainty to the overall model prediction uncertainty. Such information can guide any decisions made towards model improvement so as to enhance both accuracy and prediction confidence. The proposed methodology results in the sensitivity rankings of the various errors that contribute to the model prediction uncertainty. It is more advantageous to spend resources towards reducing an error with a higher ranking than one with a lower ranking. The sensitivity rankings are based on systematic sensitivity analysis, which is possible only after quantifying the effect of each error source on the model prediction accuracy.

The contributions of this research can be summarized as follows:

1. A systematic methodology for error and uncertainty quantification and propagation in computational mechanics models is developed. Previous literature has developed methods to quantify the discretization error, and to propagate input randomness through computational models. However, the combination of various error and uncertainty sources is not straightforward: some are additive, some multiplicative, some nonlinear, and some even nested. Also, some errors are deterministic, and some are stochastic. The methodology in this thesis provides a template to track the propagation of various error and uncertainty sources through the computational model. 
2. Sensitivity analysis methods are developed to identify the contribution of each error source to the overall uncertainty in the model prediction. Previous literature in global sensitivity analysis has only considered the effect of input random variables, and this research extends the methodology to include data uncertainty and model errors. The sensitivity information is helpful in identifying the dominant contributors to model prediction uncertainty and in guiding resource allocation for model improvement. The sensitivity analysis method is further enhanced to compare the contributions of both deterministic and stochastic errors on the same level, in order to facilitate model improvement decision making.

3. The error quantification methodology itself introduces error due to the limited number of samples; therefore a methodology is proposed in this thesis for the quantification and propagation of the UQ error. This computation includes a new nonparametric method for constructing the confidence interval for mean and variance estimated from a given set of samples.

\subsection{Organization of the thesis}

The objective of this study is to develop a methodology to quantify different errors in model prediction and investigate the importance of each error through sensitivity analysis. Chapter 2 discusses quantification and correction methods for three types of numerical errors [5]. And then a method for quantifying uncertainty quantification error 
and its propagation is proposed in Chapter 3. The quantification method of model form error is developed in Chapter 4. Chapter 5 investigates two sensitivity analysis methods for evaluating the contribution of each error. The proposed methodologies are demonstrated using two numerical examples in Chapter 6 . 


\section{CHAPTER II}

\section{NUMERICAL ERROR QUANTIFICATION}

\subsection{Introduction}

In model validation practice where the computational model is compared with the experimental results, errors are categorized into numerical error, model form error and measurement error. Quantification methodology of each kind of error is developed in this section. Especially, in model verification practice, three typical sources of the numerical error and their quantification methods are discussed, which are input error, discretization error in FEA and surrogate model error. Furthermore, a methodology for quantification and propagation of the uncertainty quantification error is proposed.

It is necessary to first distinguish between stochastic error and deterministic error before going into the following sections. In the Merriam-Webster Dictionary, the word error is defined as "the difference between an observed or calculated value and a true value”, and it appears to be a deterministic quantity. But what if the true value is unknown and we still need some estimation of the error? In this case the error can be modeled as a random variable, namely, stochastic error. Note that this does not imply that the difference between the observation or calculated value and a true value is random. 
The probability distribution only represents our belief (in a Bayesian sense) regarding the difference, or the degree of uncertainty regarding the difference, when missing the true value. The advantage of modeling the error as a random variable is that the uncertainty of the error can be easily incorporated with other uncertainty sources within a welldeveloped probability-based uncertainty quantification framework. In this research, deterministic error and stochastic error are treated differently. In order to correct a measured or calculated value, the deterministic error is directly added to it, while a random sample of the stochastic error is first generated and then added to it.

In this research, in order to quantify the numerical error, instead of comparing the original model prediction $y_{\text {pred }}$ with known solutions, it is compared with a corrected model prediction $y_{\mathrm{c}}$. Generally, if the error is deterministic, it will be corrected by adding it to its corresponding quantity; if the error is stochastic, it will be accounted for by including its uncertainty in the final output through sampling. Once the corrected model prediction $y_{\mathrm{c}}$ is obtained, the numerical error can be estimated as

$$
\varepsilon_{\text {num }}=y_{\mathrm{c}}-y_{\text {pred }}
$$

Note that since $y_{\mathrm{c}}$ and $y_{\text {pred }}$ are both considered as random variables and hence $\varepsilon_{\text {num }}$ is also a random variable, this equation is evaluated in a probabilistic sense, i.e., by sampling and substituting the samples into the equation, samples of $\varepsilon_{\text {num }}$ are obtained. In the following, all equations that involve random variables are treated the same way. 


\subsection{Input error}

In a validation exercise, the inputs to the computational model should ideally have the same values as those to the physical system. However, the inputs to the physical system model are subject to experimental variability and measurement error; therefore there is a discrepancy between what is measured or reported as the input to the physical system and its actual value. The use of this measured value in the computational model gives rise to an error in the model prediction, since the model and the actual system had different inputs.

If no prior information is available, the input errors are represented by random variables based on knowledge about experimental variability, measurement process, and instrument errors. Usually, normal distributions with mean value of zero or a constant bias are assumed. The input error can be accounted for by including its uncertainty in the final model output, i.e., by propagating the randomness of input error through the computational model. Suppose that a computational model has the form $y_{\text {pred }}=f\left(x_{1}, x_{2}, \ldots, x_{m}\right)$, in which $x_{1}, x_{2}, \ldots, x_{m}$ are model inputs and $y_{\text {pred }}$ is model prediction. Then the model output that accounts for the input errors is

$$
y_{\text {pred }}^{\prime}=f\left[x_{1}+\left(\varepsilon_{\text {in }}\right)_{1}, x_{2}+\left(\varepsilon_{\text {in }}\right)_{2}, \ldots, x_{m}+\left(\varepsilon_{\text {in }}\right)_{m}\right]
$$

where $\left(\varepsilon_{\text {in }}\right)_{1},\left(\varepsilon_{\text {in }}\right)_{2}, \ldots,\left(\varepsilon_{\text {in }}\right)_{m}$ are the (stochastic) input errors of each input variable.

\subsection{Discretization error}

Many engineering problems involve solving differential equations numerically 
using a discretization method, such as the finite difference method or finite element method. The approximation error due to coarse discretization of the domain is denoted as discretization error. The practice of quantifying the discretization error is also referred to as a posteriori error estimation in boundary value problems in mechanics. Different methods have been proposed for a posteriori error estimation, including explicit error estimation methods [12], element residual methods [13], recovery based methods [14], and extrapolation methods [15]. A comprehensive review of a posteriori error estimation is given by Ainsworth and Oden [16]. But most methods are only useful for adaptive mesh refinement, not for quantifying the actual error [1]. A good method to approximate the actual error for model V\&V is found to be the Richardson extrapolation $[17,18]$.

In this research, the Richardson extrapolation method is employed to quantify the discretization error. And we consider the estimated error is the actual error, just to demonstrate how to deal with deterministic error. However, in practice it is advised to use better methods because the Richardson Extrapolation has the following pitfalls: (1) it performs well for 1-dimensional cases, but it is less reliable after that; (2) it was first developed for finite difference method, and its result is less credible for finite element method; (3) it requires the model result to be monotonically convergent as the mesh is refined, while in most cases the result converges with fluctuation.

The Richardson Extrapolation method uses the finite element analysis as a blackbox, i.e., no other modification of the finite element code is required except refining the mesh. It requires that the finite element analysis (FEA) result be monotonically 
convergent towards the exact solution as the mesh size approaches zero. To quantify the discretization error $\varepsilon_{\mathrm{h}}$ in an FEA result $\phi_{1}$ with mesh size $h_{1}$, two more FEA runs are needed to obtained $\phi_{2}$ with a finer mesh $h_{2}$ and $\phi_{3}$ with the finest mesh $h_{3}$. Then the discretization error $\varepsilon_{\mathrm{h}}$ is given by $\varepsilon_{\mathrm{h}}=\left(\phi_{1}-\phi_{2}\right) /\left(r^{p}-1\right)$. As a result, the exact solution can be expressed as

$$
\phi_{h=0}=\phi_{1}+\left(\phi_{1}-\phi_{2}\right) /\left(r^{p}-1\right)
$$

Here $r=h_{2} / h_{1}=h_{3} / h_{2}$ is the mesh refinement ratio. And the order of convergence $p$ can be estimated as $p=\ln \left[\left(\phi_{3}-\phi_{2}\right) /\left(\phi_{2}-\phi_{1}\right)\right] / \ln (\mathrm{r})$. However, the Richardson extrapolation should be used with caution, after verifying the assumption of monotonic convergence.

\section{Example}

Consider the deflection of a slender cantilever beam with a tip load $F=3.92 \mathrm{~N}$ at the free end. The length of the beam is $L=30 \mathrm{~cm}$. The beam has a rectangular cross section of width $b=3.04 \mathrm{~cm}$ and height $h=0.078 \mathrm{~cm}$. The moment of inertia of the cross section is calculated to be $I=1.20 \times 10^{-12} \mathrm{~m}^{4}$. The Young's modulus is 200GPa and the Possion's ratio is 0.3. Ignoring the self-weight of the beam, the theoretical result for the deflection at the free end is available in [39], which is $y_{\mathrm{th}}=-12.16 \mathrm{~cm}$. This theoretical result has also been experimentally validated in [39].

A finite element model is created for the beam in ANSYS, including large 
deflection effect. The problem is solved with mesh size equal to $h_{1}=7.5 \mathrm{~cm}$, $h_{2}=3.75 \mathrm{~cm}$ and $h_{3}=1.875 \mathrm{~cm}$, respectively. The corresponding deflections are $y_{1}=-12.22 \mathrm{~cm}, y_{2}=-12.17 \mathrm{~cm}$ and $y_{3}=-12.16 \mathrm{~cm}$. The Richardson extrapolation method is used to estimate the exact solution. First the mesh refinement ratio is calculated as $r=h_{2} / h_{1}=h_{3} / h_{2}=0.5$. Then the order of convergence is calculated as $p=\ln \left[\left(\phi_{3}-\phi_{2}\right) /\left(\phi_{2}-\phi_{1}\right)\right] / \ln (\mathrm{r})=2.32$. Finally, the estimated exact solution is calculated as $y_{h=0}=y_{1}+\left(y_{1}-y_{2}\right) /\left(r^{p}-1\right)=-12.16 \mathrm{~cm}$, which matches the theoretical solution, thus illustrating the efficacy and accuracy of the Richardson extrapolation method for quantifying discretization error.

\subsection{Surrogate model prediction error}

Some engineering analyses - uncertainty quantification, sensitivity analysis, optimization etc. - require repeated runs of the finite element model, which can be prohibitively expensive. Therefore, a surrogate model, which is usually computationally much cheaper than FEA, is constructed to provide a closed-form expression of the relationship between the inputs and outputs of the original model or system. The difference between surrogate model prediction and the original model prediction is denoted as surrogate model error $\varepsilon_{\mathrm{su}}$. Since the true response of the original model is unknown at an untrained point (within the bounds of the surrogate model training), the surrogate model error is modeled as a stochastic quantity.

In this study, a polynomial chaos expansion (PCE) [33] is used as a surrogate to 
the time-consuming finite element analysis in order to generate enough Monte Carlo samples needed for quantifying the uncertainty in the model output.

PCE is a regression-based surrogate model that represents the output of a model with a series expansion in terms of standard random variables (SRVs). Consider a model $y=f(x)$ in which $y$ is the output from a numerical simulation $f(x)$ and $x=\left\{x_{1}, x_{2}, \ldots, x_{k}\right\}^{T}$ is a vector of input variables that follow certain probability distributions. Suppose PCE is constructed to replace $f(x)$ using $n$ multi-dimensional Hermite polynomials as basis functions

$$
y=\sum_{j=0}^{n} \theta_{j} \phi_{j}(\xi)=\boldsymbol{\theta}^{T} \boldsymbol{\varphi}(\xi)+\varepsilon_{\mathrm{su}}
$$

where $\xi$ is a vector of independent standard normal random variables which correspond to the original input $\mathbf{x}[21] . \varphi(\cdot)=\left\{\phi_{0}(\cdot), \phi_{1}(\cdot), \ldots, \phi_{n}(\cdot)\right\}^{T}$ are the Hermite basis functions, and $\boldsymbol{\theta}=\left\{\theta_{0}, \theta_{1}, \ldots, \theta_{n}\right\}^{T}$ are the corresponding coefficients that can be estimated by the least squares method. A collocation point method can be used to efficiently select training points where the original model is evaluated [22]. Suppose that $m$ training points $\left(\xi_{i}, y_{i}\right), i=1,2, \ldots, m$ are available. Under the Gauss-Markov assumption [23], the surrogate model error $\varepsilon_{\text {su }}$ asymptotically follows a normal distribution with zero mean and variance given by

$$
\operatorname{Var}\left[\varepsilon_{\mathrm{su}}\right] \approx s^{2}+s^{2} \boldsymbol{\varphi}(\xi)^{T}\left(\boldsymbol{\Phi}^{T} \boldsymbol{\Phi}\right)^{-1} \boldsymbol{\varphi}(\xi)
$$

where $\boldsymbol{\Phi}=\left\{\boldsymbol{\varphi}\left(\boldsymbol{\xi}_{1}\right), \boldsymbol{\varphi}\left(\boldsymbol{\xi}_{2}\right), \ldots, \boldsymbol{\varphi}\left(\boldsymbol{\xi}_{m}\right)\right\}^{T}$ and $s^{2}=\frac{1}{m-n} \sum_{i=1}^{m}\left[y_{i}-\boldsymbol{\theta}^{T} \boldsymbol{\varphi}\left(\boldsymbol{\xi}_{i}\right)\right]^{2}$. 
In order to account for the surrogate model error, random samples of $\varepsilon_{\text {su }}$ are generated and added to the surrogate model prediction. As a result, instead of only one, a number of sample values of surrogate model predictions are obtained for a single input, and all sample values should be used for succeeding analyses and obtain a number of sample values of final model prediction. This approach to account for the surrogate model prediction error also works for other stochastic surrogate models such as Gaussian Process model [24] that provide the uncertainty, namely, the variance, of prediction.

\section{Example}

Consider the slender cantilever beam problem described in Section 2.3. Assume the concentrated force follows a normal distribution with mean $3.5 \mathrm{~N}$ and standard deviation 0.4 N. 15 samples of $\mathrm{F}$ are generated and 15 corresponding beam tip deflections are calculated using the finite element model with mesh size $h=7.5 \mathrm{~cm}$. The results are listed in Table 1. The first 9 results are used to construct a first order PCE surrogate model, and the last 6 are used as validation points. The PCE model is then used to predict the beam tip deflection over the range of force from $3 \mathrm{~N}$ to $4.5 \mathrm{~N}$. The error associated with each prediction is calculated based on which 90\% confidence bounds are constructed. The results are plotted in Figure 1, and show excellent agreement between the surrogate model prediction and the original FEA predictions at the validation points. 
Table 1. 15 FEA results of the slender cantilever beam problem

\begin{tabular}{|c|c|c|}
\hline & Force (N) & Deflection (cm) \\
\hline \multirow{4}{*}{ Training } & 3.13 & -10.33 \\
\cline { 2 - 3 } points & 3.47 & -11.18 \\
\cline { 2 - 3 } & 3.59 & -11.47 \\
\cline { 2 - 3 } & 3.63 & -11.56 \\
\cline { 2 - 3 } & 3.71 & -11.75 \\
\cline { 2 - 3 } & 3.74 & -11.82 \\
\cline { 2 - 3 } & 3.90 & -12.18 \\
\cline { 2 - 3 } & 4.12 & -12.65 \\
\cline { 2 - 3 } & 4.35 & -13.13 \\
\hline \multirow{4}{*}{ Validation } & 3.08 & -10.20 \\
\cline { 2 - 3 } points & 3.20 & -10.51 \\
\cline { 2 - 3 } & 3.40 & -11.01 \\
\cline { 2 - 3 } & 3.53 & -11.33 \\
\cline { 2 - 3 } & 3.67 & -11.66 \\
\cline { 2 - 3 } & 3.96 & -12.31 \\
\hline
\end{tabular}

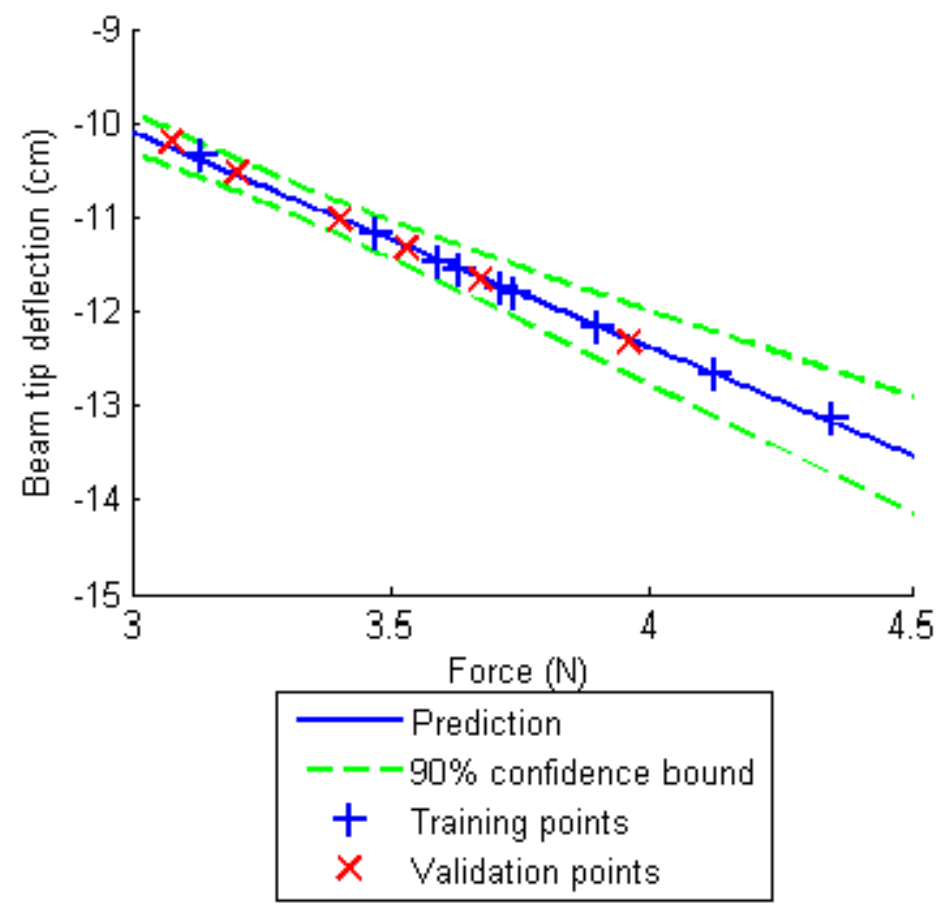

Figure 1. Surrogate model prediction and confidence bounds 


\section{CHAPTER III}

\section{UNCERTAINTY QUANTIFICATION ERROR}

\subsection{Introduction}

In this chapter, we discuss the error which arises when using a limited number of samples to estimate the mean and variance or construct the empirical CDF of a stochastic quantity. We denote the uncertainty quantification error $\varepsilon_{\text {uq }}$ as the difference between the empirical CDF value and the "true" CDF value. First an error estimation method is developed to quantify $\varepsilon_{\text {uq }}$. Then based on this a method is developed for interval estimation of mean and variance. Furthermore, the propagation of $\varepsilon_{\text {uq }}$ is studied when resampling from the empirical CDF and using the samples for further analysis.

\subsection{Error in Empirical CDF}

Suppose that an empirical CDF $\bar{F}_{X}(x)$ is constructed from $N_{\mathrm{s}}$ samples of a random variable $X$. And $F_{X}(x)$ is referred to as the CDF value that includes the error in the empirical CDF. Suppose that $n$ samples are smaller than a specific value $x$; then $n$ should follow a binomial distribution [34] $n \sim B\left(N_{\mathrm{s}}, \bar{F}_{X}(x)\right)$ if we consider that the value of each sample is a result of Bernoulli trial. The binomial distribution approaches the 
normal distribution as $N_{\mathrm{s}}$ increases. When $N_{\mathrm{s}}$ is greater than 20, the normal distribution $n \sim N\left(N_{\mathrm{s}} \bar{F}_{X}(x), \sqrt{N_{\mathrm{s}} \bar{F}_{X}(x)\left[1-\bar{F}_{X}(x)\right]}\right)$ is a good approximation to the original binomial distribution. Since $F_{X}(x)=n / N_{\mathrm{s}}$, we have

$$
F_{X}(x)=\frac{n}{N_{\mathrm{s}}} \sim N\left(\bar{F}_{X}(x), \sqrt{\frac{\bar{F}_{X}(x)\left[1-\bar{F}_{X}(x)\right]}{N_{\mathrm{s}}}}\right)
$$

Therefore $\varepsilon_{\text {uq }}$, the error associated with the CDF value $\bar{F}_{X}(x)$ can be represented as a normal random variable with zero mean and variance $\sigma^{2}$, which can be estimated from:

$$
\sigma^{2}=\frac{\left[1-\bar{F}_{X}(x)\right] \bar{F}_{X}(x)}{N_{\mathrm{s}}}
$$

in which $N$ is the number of samples used to construct the empirical CDF of $x$. Note that the variance of this error is actually a function of $x$, and it goes to zero at both ends of the CDF curve. Or we can directly treat $F_{X}(x)$ as a random variable with variance $\sigma^{2}$

\section{Example}

Suppose $X \sim N(0,1)$ and 21 samples of $X$ are available. The empirical CDF of the samples with $90 \%$ confidence bounds are constructed using the above method. The result is compared to the true CDF curve in Figure 2, and show that the CDF curve lies between the confidence bounds. 


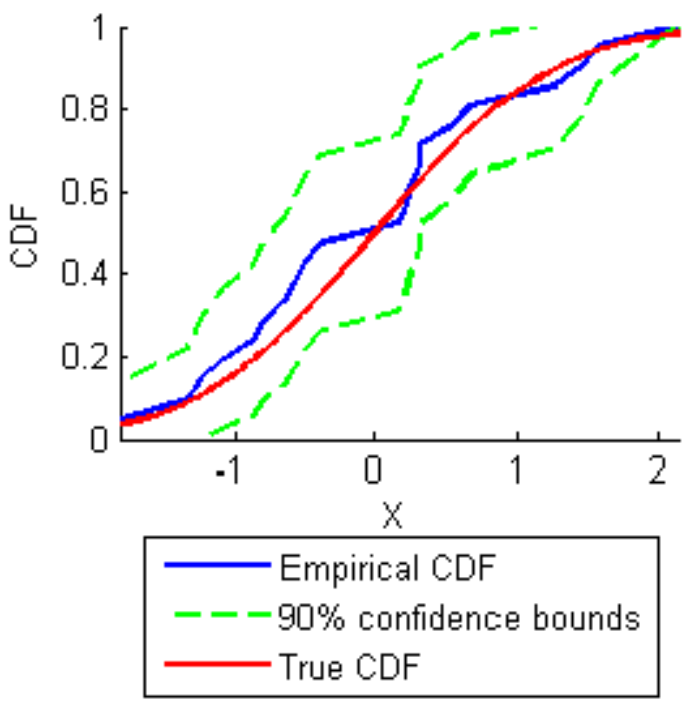

Figure 2. Empirical CDF with 90\% confidence bounds vs. true CDF

\subsection{Error Estimation for mean and variance}

The classical interval estimation method for mean and variance from samples of a normal variable is available in most statistics texts [34]. Recently, methods were developed to handle non-normal cases [35, 36]. Here we propose an estimation method for mean and variance, based on the error estimation for CDF established above. The mean and variance are modeled as random variables and their samples are obtained by directly integrating the empirical CDF while considering the random error in the CDF value. Then the samples of the mean and variance can be used to construct their confidence intervals. This method can be applied without knowing the specific distribution type of the samples. Suppose $N_{\mathrm{s}}$ samples of $x$ are given, then by definition, the estimated mean is can be obtained by 


$$
\mu_{X}=E(X)=\int_{-\infty}^{+\infty} x f_{X}(x) d x
$$

where $f_{X}(x)$ is the PDF of $x$. According to the range of samples, the integration interval can be replaced by one with reasonable upper and lower bound, i.e., $\left[x_{l}, x_{u}\right]$, such that $F_{X}\left(x_{l}\right)=0$ and $F_{X}\left(x_{u}\right)=1$. Note the relationship between PDF and CDF, we have

$$
\mu_{X}=\int_{x_{l}}^{x_{u}} x \frac{d F_{X}(x)}{d x} d x=\int_{x_{l}}^{x_{u}} x d F_{X}(x)
$$

Integrating by parts gives

$$
\mu_{X}=\left.x F_{X}(x)\right|_{x_{l}} ^{x_{u}}-\int_{x_{l}}^{x_{u}} F_{X}(x) d x=x_{u}-\int_{x_{l}}^{x_{u}} F_{X}(x) d x
$$

The integration term can be approximated by summation, which yields

$$
\mu_{X}=x_{u}-\sum_{i} F_{X}\left(x_{i}\right) \Delta x_{i}
$$

The summation term can be evaluated by sampling $F_{X}\left(x_{i}\right)$. The partitions $x_{i}$ should be the same as those when $F_{X}(x)$ is constructed. By repeating this process, samples of $\mu_{X}$ are obtained.

Similarly we can estimate $\sigma_{X}^{2}$ :

$$
\begin{aligned}
\sigma_{X}^{2} & =\operatorname{Var}[X]=E\left[X^{2}\right]-E[X]^{2} \\
& =\int_{-\infty}^{+\infty} x^{2} f_{X}(x) d x-\mu_{X}^{2} \\
& =\int_{x_{l}}^{x_{u}} x^{2} d F_{X}(x)-\mu_{X}^{2} \\
& =\left.x^{2} F_{X}(x)\right|_{x_{l}} ^{x_{u}}-\int_{x_{l}}^{x_{u}} F_{X}(x) \cdot 2 x d x-\mu_{X}^{2} \\
& =x_{u}^{2}-2 \sum_{i} x_{i} F_{X}\left(x_{i}\right) \Delta x_{i}-\mu_{X}^{2}
\end{aligned}
$$

Substituting $\mu_{X}$ from Eq. (3.6) into Eq. (3.7) gives: 


$$
\begin{aligned}
\sigma_{X}^{2} & =x_{u}^{2}-2 \sum_{i} x_{i} F_{X}\left(x_{i}\right) \Delta x_{i}-\left(x_{u}-\sum_{i} F_{X}\left(x_{i}\right) \Delta x_{i}\right)^{2} \\
& =x_{u}^{2}-2 \sum_{i} x_{i} F_{X}\left(x_{i}\right) \Delta x_{i}-x_{u}^{2}+2 x_{u} \sum_{i} F_{X}\left(x_{i}\right) \Delta x_{i}-\left(\sum_{i} F_{X}\left(x_{i}\right) \Delta x_{i}\right)^{2} \\
& =2 \sum_{i}\left(x_{u}-x_{i}\right) F_{X}\left(x_{i}\right) \Delta x_{i}-\left[\sum_{i} F_{X}\left(x_{i}\right) \Delta x_{i}\right]^{2}
\end{aligned}
$$

Again, by sampling the summation term, samples of $\sigma_{X}^{2}$ are obtained. Notice that $F_{X}\left(x_{i}\right)$ should be the same in both terms (use the same sample), i.e., they should have the same value for a given $x_{i}$.

\section{Example}

An example is shown here to demonstrate how to use the proposed method to estimate the true mean and variance of given samples and how to construct confidence intervals for the estimates. In order to compare with the classical statistics method, we assume that $n=21$ samples of a normal random variable $X$ are available but its variance is unknown. The true mean and variance of $X$ are denoted as $\mu_{X}$ and $\sigma_{X}^{2}$, which are both unknown.. And the sample mean and variance are estimated from the samples: $\bar{X}=5.14$ and $S^{2}=10.75$. From basic statistics, we know that $\frac{\bar{X}-\mu_{X}}{S / \sqrt{n}}$ follows a tdistribution with $n-1=20$ degrees of freedom; and $\frac{(n-1) S^{2}}{\sigma_{X}^{2}}$ follows a chi-square distribution with $n-1=20$ degree of freedom. The PDFs of $\mu_{X}$ and $\sigma_{X}^{2}$ are plotted in Figure 3 (a) and (b) respectively. And the $90 \%$ confidence intervals of both $\mu_{X}$ and $\sigma_{X}^{2}$ are $\left\langle\mu_{X}>_{0.90}=[3.90,6.37]\right.$ and $\left\langle\sigma_{X}^{2}>_{0.90}=[6.84,19.81]\right.$. 
Using the proposed method, 1000 samples for each of $\mu_{X}$ and $\sigma_{X}^{2}$ are obtained using Eqs. (3.6) and (3.8), respectively. Kernel smoothing density estimates of the samples are computed for $\mu_{X}$ and $\sigma_{X}^{2}$, respectively, and the results are also plotted in Figure 3. Comparing with the classical method, the PDFs of $\sigma_{x}^{2}$ agree quite well, while the PDF of $\mu_{X}$ given by the proposed method is narrower than that given by the classical method. From the samples, the $90 \%$ confidence intervals of $\mu_{X}$ and $\sigma_{X}^{2}$ are calculated to be $\left\langle\mu_{X}\right\rangle_{0.90}=[4.60,5.66]$ and $\left\langle\sigma_{X}^{2}\right\rangle_{0.90}=[6.06,14.70]$. Compared to the results given by the classical method, the proposed method gives a narrower estimation for the mean.
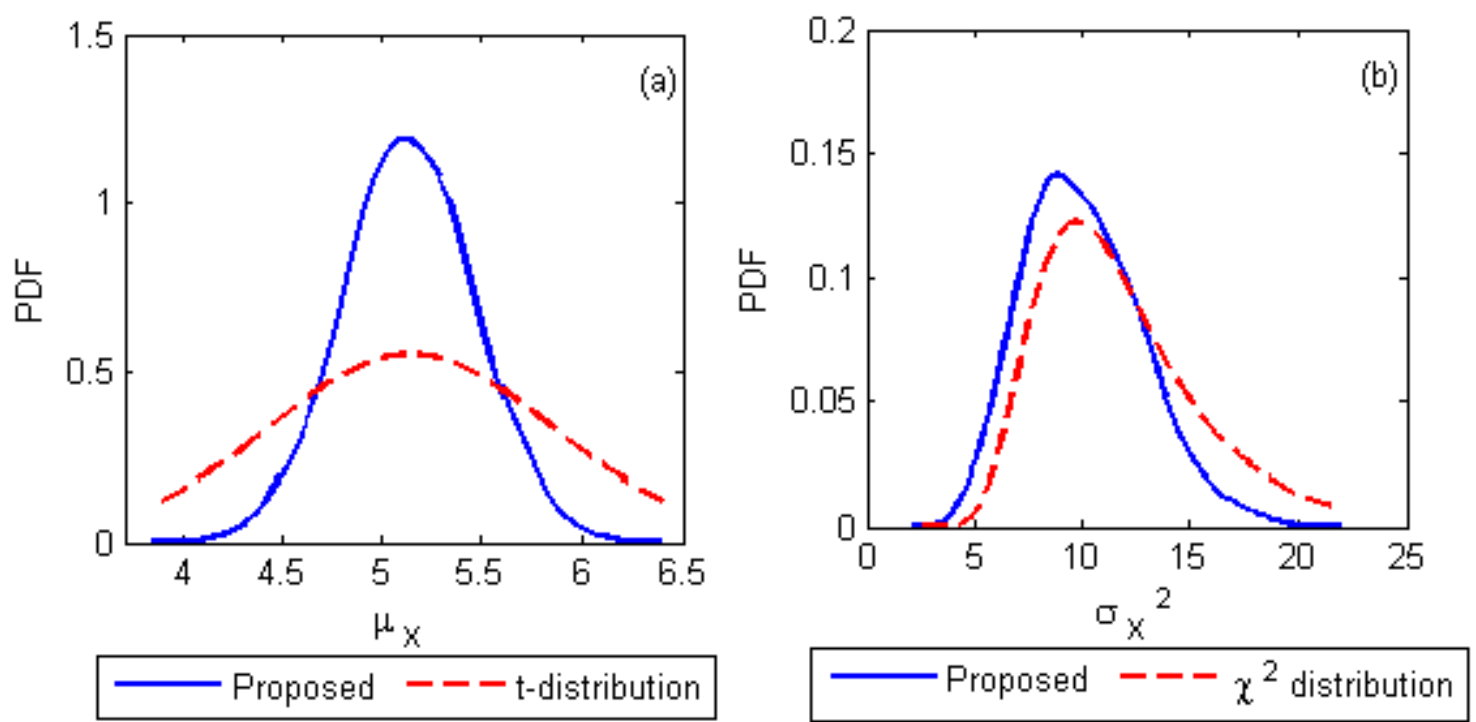

Figure 3. (a) PDF of $\mu_{X}$; (b) PDF of $\sigma_{X}^{2}$ 


\subsection{Propagation of UQ error}

If more realizations of $x$ are generated from the empirical CDF to calculate the distribution of another variable $y=g(x)$, then $\varepsilon_{\text {uq }}$ is propagated through the sampling procedure and results in sampling error $\varepsilon_{\mathrm{s}}$ in the new samples of $x$. If an inverse CDF method is used for sampling, an approximate method for quantifying the sampling error $\varepsilon_{\mathrm{s}}$ is proposed below.

To generate the ith sample $x_{i}$, a random number $u_{i}$ is first generated from a uniform distribution $u \sim U(0,1)$. By inverting $u=F_{x}(x)$, a sample $x_{i}$ is obtained $\left(F_{x}(x)\right.$ is the empirical CDF). However, due to the error $\left(\varepsilon_{\text {uq }}\right)_{i}$ in the CDF value, the actual CDF curve might be along the dotted line as shown in Figure 4. As a result, the actual sample value we should get can be expressed as $x_{i}+\left(\varepsilon_{\mathrm{s}}\right)_{i}$, where $\left(\varepsilon_{\mathrm{s}}\right)_{i}$ is the sampling error of $x_{i}$. Since $\left(\varepsilon_{\text {uq }}\right)_{i}$ reflects how much the empirical CDF deviates from the actual CDF at $x=x_{i}$ and in its neighborhood, $\left(\varepsilon_{\mathrm{s}}\right)_{i}$ should increase as $\left(\varepsilon_{\mathrm{uq}}\right)_{i}$ increases. Especially, $\left(\varepsilon_{\mathrm{s}}\right)_{i}$ should tend to zero when $\left(\varepsilon_{\text {uq }}\right)_{i}$ is equal to zero. In Figure 4 , let $\left(\varepsilon_{\text {uq }}\right)_{i}$ and $\left(\varepsilon_{\mathrm{uq}}\right)_{i}{ }^{\prime}$ be the errors corresponding to the CDF values at $x_{i}$ and $\left[x_{i}+\left(\varepsilon_{\mathrm{s}}\right)_{i}\right]$, respectively. From Eqs. (3.1) and (3.2), we know that $\left(\varepsilon_{\mathrm{uq}}\right)_{i} \sim N\left[0, \sigma\left(x_{i}\right)\right]$ and $\left(\varepsilon_{\text {uq }}\right)_{i}{ }^{\prime} \sim N\left(0, \sigma\left[x_{i}+\left(\varepsilon_{\mathrm{s}}\right)_{i}\right]\right)$. If we let $\left(\varepsilon_{\text {uq }}\right)_{i}$ and $\left(\varepsilon_{\text {uq }}\right)_{i}{ }^{\prime}$ have the same percentile value (assuming $\left(\varepsilon_{\mathrm{s}}\right)_{i}$ is very small), then we have

$$
\Phi\left[\frac{\left(\varepsilon_{\mathrm{uq}}\right)_{i}-0}{\sigma\left(x_{i}\right)}\right]=\Phi\left[\frac{\left(\varepsilon_{\mathrm{uq}}\right)_{i}{ }^{\prime}-0}{\sigma\left[x_{i}+\left(\varepsilon_{\mathrm{s}}\right)_{i}\right]}\right]=\Phi\left[\frac{u_{i}-F_{x}\left[x_{i}+\left(\varepsilon_{\mathrm{s}}\right)_{i}\right]}{\sigma\left[x_{i}+\left(\varepsilon_{\mathrm{s}}\right)_{i}\right]}\right]
$$

In this equation $\left(\varepsilon_{\mathrm{s}}\right)_{i}$ is the unknown quantity to be estimated. Based on this 
assumption a one-to-one mapping relationship is established between $\left(\varepsilon_{\mathrm{s}}\right)_{i}$ and $\left(\varepsilon_{\text {uq }}\right)_{i}$ and therefore one can calculate $\left(\varepsilon_{\mathrm{s}}\right)_{i}$ for a given $\left(\varepsilon_{\mathrm{uq}}\right)_{i}$. Since the distribution of $\left(\varepsilon_{\mathrm{uq}}\right)_{i}$ is assumed in Section 3.2, one can obtain realizations of $\left(\varepsilon_{\mathrm{s}}\right)_{i}$ by sampling $\left(\varepsilon_{\mathrm{uq}}\right)_{i}$. Thus the corrected value of $x_{i}$ is $\left[x_{i}+\left(\varepsilon_{s}\right)_{i}\right]$, which includes the sampling error $\left(\varepsilon_{s}\right)_{i}$. Now $y=g(x)$ is calculated using the corrected realization.

This method offers a generic approach to resample from sparse data, and include the extra uncertainty due to UQ error into the new samples. In this research, this method is later applied to quantify model form error (see Section 4). A kernel density estimation may be performed to obtained a smoothed empirical CDF before applying this method, if necessary.

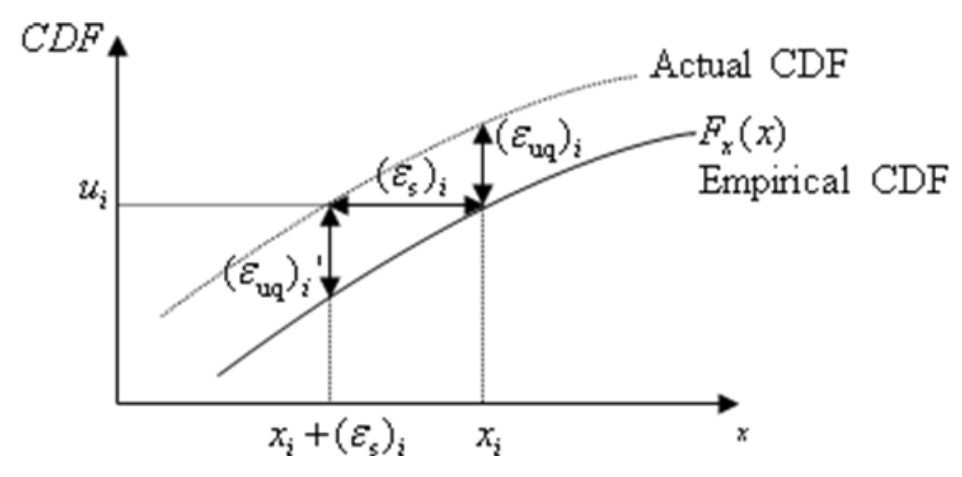

Figure 4. Sampling error quantification

\subsection{Summary}

Using limited number of samples in sampling-based uncertainty quantification creates uncertainty quantification error (UQ error). This chapter defines the UQ error as 
the error that arises in the value of the empirical CDF constructed from the samples, and a methodology to quantify this error is developed. Based on this, a method is proposed to compute the confidence intervals of the mean and variance estimates from the given samples. This method is nonparametric and can be applied to samples of arbitrary distribution. For a special case where the samples come from an underlying normal distribution, the result given by this method is compared to that available from classic statistics. The results for variance estimate agree quite well but proposed method gives a narrower distribution of mean estimate than classical method. More work must be done to include the correlation between different values of $F_{X}\left(x_{i}\right)$. Furthermore, a method is developed to resample from the empirical CDF considering the UQ error, in generating more samples for uncertainty propagation analysis. 


\section{CHAPTER IV}

\section{MODEL FORM ERROR ESTIMATION}

\subsection{Introduction}

Model form error is independent of the numerical solution error. As mentioned in Section 1.1, quantification of model form error requires comparison of model prediction against validation experiment observations $y_{\mathrm{obs}}$, where output measurement error $\varepsilon_{\mathrm{om}}$ needs to be taken into account. Using equating Eqs. (1.1) and (1.2), we have

$$
\underbrace{y_{\text {pred }}+\varepsilon_{\text {num }}}_{y_{\mathrm{c}}}+\varepsilon_{\text {model }}=y_{\text {obs }}+\varepsilon_{\text {om }}
$$

Rearranging the terms gives:

$$
\varepsilon_{\text {model }}=y_{\text {obs }}+\varepsilon_{\text {om }}-y_{\mathrm{c}}
$$

This equation is evaluated by sampling each of the three terms on the right-hand side and samples of $\varepsilon_{\text {model }}$ are obtained. Quantification of the output measurement error $\varepsilon_{\text {om }}$ is discussed in the next section. Furthermore, UQ error arises when calculating the model form error since a sampling-based method is used. Section 4.3 discusses how to take the UQ error into account when calculating model form error. 


\subsection{Output measurement error}

Output measurement error arises here when measuring the output of the physical system. This comes into the picture in model validation and model form error quantification. The output measurement error is commonly assumed to be a Gaussian random variable with zero mean and an assumed variance [23], based on measurement process and equipment.

\subsection{Model form error}

In most cases only the distribution of $\varepsilon_{\text {om }}$ might be available, and only a few point-valued data are available for $y_{\mathrm{obs}}$. Moreover, since the procedure of computing $y_{\mathrm{c}}$ requires considerable computational effort, then only limited number of samples can be

obtained. A re-sampling approach is needed to generate more samples of $y_{\mathrm{obs}}$ and $y_{\mathrm{c}}$ from sparse data, but re-sampling could also cause another error. Therefore, the approach developed in Section 3.4 for re-sampling and quantifying the sampling error is used. Then Eq. (4.2) becomes

$$
\varepsilon_{\text {model }}=\left[y_{\text {obs }}+\left(\varepsilon_{\mathrm{s}}\right)_{\mathrm{obs}}\right]+\varepsilon_{\mathrm{om}}-\left[y_{\mathrm{c}}+\left(\varepsilon_{\mathrm{s}}\right)_{\mathrm{c}}\right]
$$

It is clear that the error $\varepsilon_{\mathrm{s}}$ also contributes to the model form error.

\subsection{Summary}

Once the model form error statistics are calculated using Eq. (4.3), for future prediction purposes, the overall corrected prediction that includes both solution 
approximation error and model form error may be computed as $y_{\mathrm{c}}+\varepsilon_{\text {model }}$. In this expression, $y_{c}$ includes the contribution of various solution approximation errors and input error, and $\varepsilon_{\text {model }}$ includes the contribution of UQ error and output measurement error. Of course, the UQ error is not an independent error source; it only arises due to the limited number of samples used in the analysis, and can be diminished by increasing the number of samples. 


\section{CHAPTER V}

\section{SENSITIVITY ANALYSIS}

\section{$5.1 \quad$ Introduction}

The previous chapters developed a methodology to quantify different errors in model prediction. In this chapter, sensitivity analysis is performed to estimate the contribution of each error source to the model prediction uncertainty. Previous studies in stochastic sensitivity analysis have only considered the effect of input random variables; this research extends those methods to include sources of solution approximation error. The sensitivity analysis is done in terms of the contribution to uncertainty in the corrected model prediction $y_{c}$ (defined in Eq. (2.1)). Since the UQ error and model form error are computed only after the computation of $y_{c}$, this sensitivity analysis is only with respect to solution approximation errors, and does not include UQ error or model form error.

In this chapter, two local and two global sensitivity measures are studied. In order to perform sensitivity analysis a model output that considers all errors is needed, which is $y_{\text {c }}$ (see Chapter 2). For the sake of demonstration, a generic model $Y=f\left(X_{1}, X_{2}, \ldots, X_{k}\right)$ is assumed, where $X_{i}$ can be either model input, error or model parameter with uncertainty. 


\subsection{Local sensitivity analysis}

\subsubsection{Change in variance}

The change of variance in the model prediction due to the $i$ th input is defined as:

$$
\left(\Delta \sigma^{2}\right)_{i}=\operatorname{Var}(Y)-\operatorname{Var}_{X_{\aleph_{i}}}\left(Y \mid X_{i}=\bar{x}_{i}\right)
$$

This equation provides a measure of the change in variance of $Y$ if the ith input is ignored. Ignoring $X_{i}$ means fixing it at its mean value (usually zero for an error term). The second term in right hand side of Eq. (5.1) is a conditional variance of $Y$ given $X_{i}$, and is taken over $X_{\sim i}$ (all $X$ but $X_{i}$ ). The greater $\left(\Delta \sigma^{2}\right)_{i}$ is, the more important $X_{i}$ is. However, this measure is only in terms of variance and ignores other uncertainty information such as mean, skewness, kurtosis etc, Also, the result depends on where $X_{i}$ is fixed, and in occasional cases, it is possible that $\operatorname{Var}_{X_{\sim i}}\left(Y \mid X_{i}=\bar{x}_{i}\right)>\operatorname{Var}(Y)$. For example, consider a model $Y=X_{1} \cdot X_{2}$ with two inputs $X_{1} \sim N(0,1)$ and $X_{2} \sim N(0,1)$. It is easy to verify that $\operatorname{Var}\left(Y \mid X_{1}=2\right)=4>\operatorname{Var}(Y)=1$. But due to its simplicity, change of variance $\left(\Delta \sigma^{2}\right)_{i}$ is still an applicable scalar measurement of the effect due to each contributing source of uncertainty. Since $X_{i}$ is fixed at a single value, the change of variance is a local sensitivity measure.

\subsubsection{Kullback-Leibler divergence}

The Kullback-Leibler divergence (K-L divergence) [30], adopted from information theory, measures the difference between two probability density functions 
$p(x)$ and $q(x)$, in the sense of the relative information entropy (uncertainty) of $p(x)$ with respect to $q(x)$. It is defined by

$$
D_{\mathrm{KL}}(P \| Q)=\int_{-\infty}^{\infty} p(x) \log \frac{p(x)}{q(x)} d x
$$

The K-L divergence is nonnegative, and it is zero if and only if $p(x)$ and $q(x)$ are exactly the same. As illustrated in Figure 5, the area to be integrated clearly reflects the difference in shape between $p(x)$ and $q(x)$. It is sensitive to both differences in mean value and in variance.
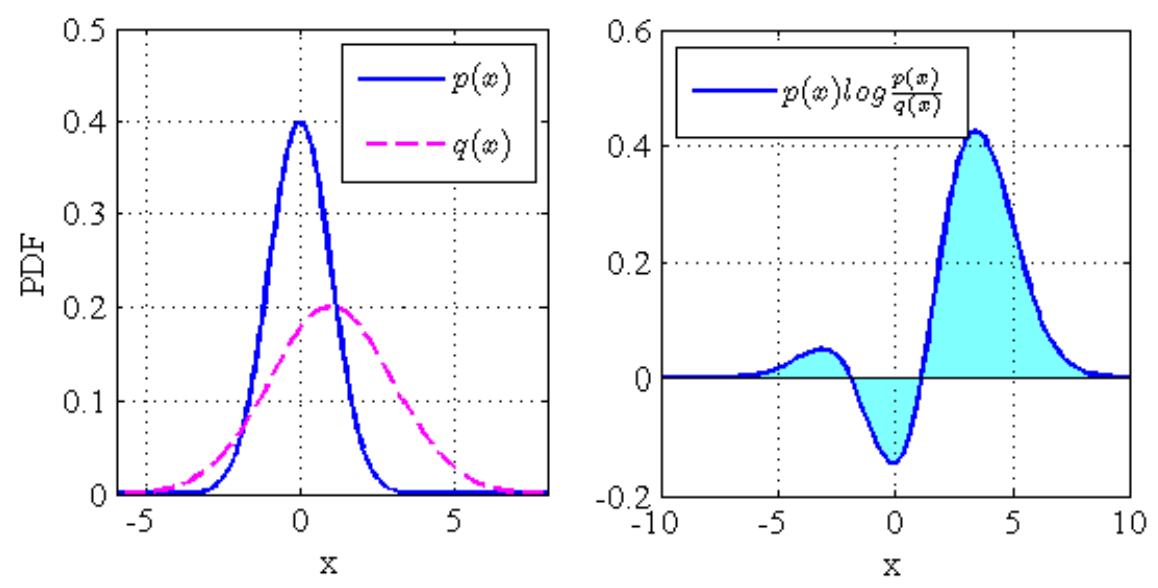

Figure 5. Illustration of K-L divergence $D_{\mathrm{KL}}(P \| Q)$, which is equal to the integral of the showed area in the left figure.

The K-L divergence has been used in sensitivity analysis [27] to measure the contribution of an individual source to the uncertainty in the model prediction. In order to compare with the measure of change of variance, the K-L divergence is used to assess the 
total sensitivity of $Y$ to $X_{i}$, which is given by $D_{\mathrm{KL} X_{i}}\left[\left(Y \mid X_{i}=\bar{x}_{i}\right) \| Y\right]$. This compares the difference over the entire distributions of $Y$ and the conditional $\left(Y \mid X_{i}=\bar{x}_{i}\right)$, in which $X_{i}$ is fixed at a value (usually its mean). A larger value of the $\mathrm{KL}$ divergence means $Y$ is more sensitive to $X_{i}$.

\subsection{Global sensitivity analysis}

\subsubsection{Main effect and total effect measures}

The drawbacks of change of variance motivate the need for a better sensitivity measure. Instead of fixing $X_{i}$ at a single value, we can average the conditional variance $\operatorname{Var}_{X_{\sim_{i}}}\left(Y \mid X_{i}\right)$ over the entire distribution of $X_{i}$. This is denoted as $E_{X_{i}}\left[\operatorname{Var}_{X_{\triangle i}}\left(Y \mid X_{i}\right)\right]$, and it no longer depends on the where $X_{i}$ is fixed, so that it is "global" over the range of $X_{i}$. Starting with this and based on variance decomposition [26], the variance of $Y$ can be decomposed into two terms, with respect of $X_{i}$ [37],

$$
\operatorname{Var}(Y)=E_{X_{i}}\left[\operatorname{Var}_{X_{N_{i}}}\left(Y \mid X_{i}\right)\right]+\operatorname{Var}_{X_{i}}\left[E_{X_{N_{i}}}\left(Y \mid X_{i}\right)\right]
$$

Here both terms are complimentary of each other. Either a smaller first term or a bigger second term indicates a more important $X_{i}$. By normalizing the second we obtain the main effect sensitivity index:

$$
S_{i}=\frac{\operatorname{Var}_{X_{i}}\left[E_{X_{\wedge i}}\left(Y \mid X_{i}\right)\right]}{\operatorname{Var}(Y)}
$$

which is always between 0 and 1 . The main effect is also referred to as first-order effect. Note that a low main effect index does not imply that the variable is not important. A 
variable with a low main effect might make a bigger contribution to the model output through interaction with other variables. Therefore a more comprehensive sensitivity measure which includes the main effect and the interaction effect is needed, which is the total effect index. If we swap $X_{i}$ and $X_{\sim i}$ in Eq. (5.3), another way of decomposing the variance of $Y$ is discovered:

$$
\operatorname{Var}(Y)=E_{X_{\alpha_{i}}}\left[\operatorname{Var}_{X_{i}}\left(Y \mid X_{\sim i}\right)\right]+\operatorname{Var}_{X_{\alpha_{i}}}\left[E_{X_{i}}\left(Y \mid X_{\sim i}\right)\right]
$$

By normalizing the first term we obtain the total effect sensitivity index:

$$
S_{\mathrm{T}_{\mathrm{i}}}=\frac{E_{X_{\sim_{i}}}\left[\operatorname{Var}_{X_{i}}\left(Y \mid X_{\sim i}\right)\right]}{\operatorname{Var}(Y)}
$$

The total effect index becomes valuable when the sum of individual main effect indices is not close to 1 , which implies that strong interaction effects exist among variables. Since the total effect index accounts for the total contribution to the output due to the input $X_{i}$, the condition $S_{\mathrm{T}_{\mathrm{i}}}=0$ is a necessary and sufficient condition for $X_{i}$ being negligible, i.e., fixing $X_{i}$ at a particular value has almost no influence to the model output.

By brute force, evaluation of both $\operatorname{Var}_{X_{i}}\left[E_{X_{\sim_{i}}}\left(Y \mid X_{i}\right)\right]$ and $E_{X_{\sim_{i}}}\left[\operatorname{Var}_{X_{i}}\left(Y \mid X_{\sim i}\right)\right]$ request a nest-loop Monte Carlo simulation. If $N$ samples are needed to calculate both the expectation and the variance, then totally $N \times N$ runs of computing the model output $Y$ are needed. To accelerate the process, an efficient method was developed in [38].

\subsection{An intuitive understanding of the sensitivity measures}

Scatter plots can be used to help explain the above sensitivity measures, except 
for total effect index, which cannot be illustrated in a 2-D plot. Suppose that adequate samples of $Y$ are generated by Monte Carlo simulation; the scatter plot of $Y$ versus $X_{i}$ is shown in Figure 6(a). In this figure, consider a thin strip centered at the mean value of $X_{i}$. If the strip is thin enough, the samples that fall within the strip could be considered as the samples of $\left(Y \mid X_{i}=\bar{x}_{i}\right)$. Actually, the change of variance measure compares the variance of the samples that fall within the strip vs. the variance of all samples, both with respect to $Y$. If PDFs are constructed for the samples of both $Y$ and $\left(Y \mid X_{i}=\bar{x}_{i}\right)$, then the KL divergence measure can be calculated by comparing the two distributions.

Similarly, we can calculate the mean value of the samples in the strip, which is given by $E_{X_{\sim i}}\left(Y \mid X_{i}=\bar{x}_{i}\right)$. Then if the position of the strip varies, the samples of $E_{X_{\sim_{i}}}\left(Y \mid X_{i}\right)$ are obtained as shown in Figure 6(c). Finally if the position of the strip varies according to the distribution of $X_{i}$ (as shown in Figure 6(b)) then we can calculate the variance of $E_{X_{c i}}\left(Y \mid X_{i}\right)$ with respect to $X_{i}$, which is the main effect $\operatorname{Var}_{X_{i}}\left[E_{X_{x_{i}}}\left(Y \mid X_{i}\right)\right]$

However, none of the above sensitivity measures is perfect. Change of variance measures the local sensitivity at a particular $X_{i}$, and only addresses the variance but ignores other information. The main effect and total effect sensitivity index is "global" over the entire range of $X_{i}$, but again it addresses only the variance. The KL divergence compares the entire distribution which makes it "global" with respect to $Y$, but it is still local with respect to $X_{i}$ since it is calculated at a particular value of $X_{i}$. 

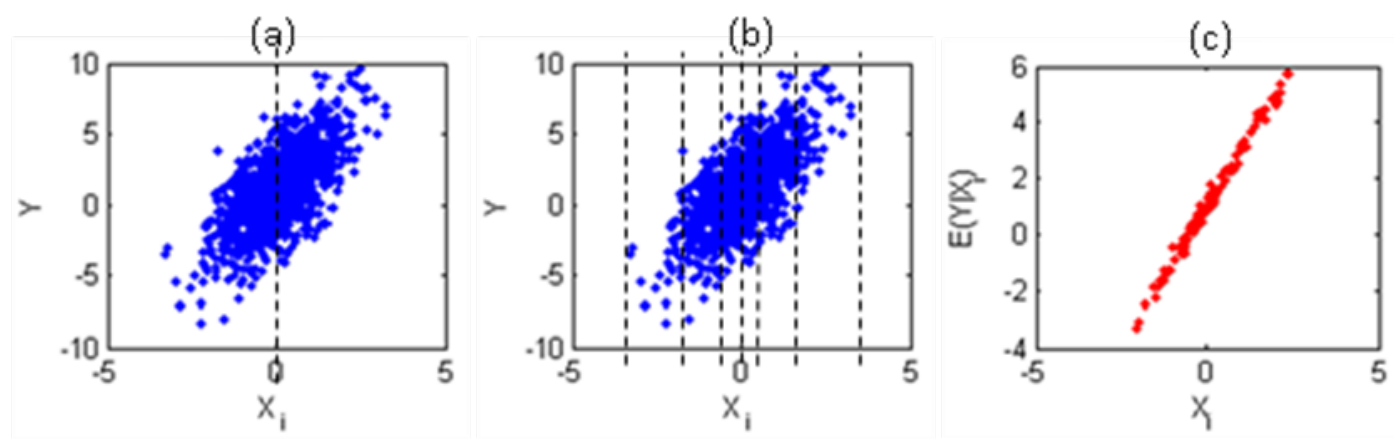

Figure 6. Scatter plots: (a) $Y$ versus $X_{i}$ with a slice cut at $X_{i}=0$; (b) $Y$ versus $X_{i}$ with slices cut at different $X_{i}$; (c) $E_{X_{\sim i}}\left(Y \mid X_{i}\right)$ versus $X_{i}$.

\subsection{Deterministic and stochastic errors in sensitivity analysis}

It was mentioned in Section 1.1 that deterministic errors (such as discretization error) are corrected, whereas stochastic errors are accounted for through sampling, in order to compute the corrected model prediction $y_{\mathrm{c}}$. In this case, only local sensitivity measures can be calculated corresponding to deterministic errors, which obviously have only fixed values for fixed model inputs. The corrected model prediction is calculated with and without correcting for the deterministic error, and the corresponding change in variance or K-L distance can be computed.

For stochastic errors, it is more appropriate compute global sensitivity measures instead of at one particular value (typically the mean). However, this creates a difficulty in comparing the relative contributions of the deterministic vs. stochastic errors to the overall model prediction uncertainty, when resource allocation decisions are needed for 
activities such as model refinement and data collection. Therefore, an approximate approach is proposed below to compute global sensitivity measures for even deterministic errors.

In global sensitivity analysis with stochastic variables, samples of the variables are generated based on their distributions. But deterministic variables do not have a distribution to sample from, such as discretization error. An approximate approach is to obtain samples of $\varepsilon_{\mathrm{h}}$ corresponding to samples of random inputs to the FEA model; these samples of $\varepsilon_{\mathrm{h}}$ are used to construct the distribution of $\varepsilon_{\mathrm{h}}$.

Another interesting problem occurs in calculating global sensivity measures with respect to the surrogate model error $\varepsilon_{\text {su }}$, even it is stochastic. This is because the distribution of $\varepsilon_{\text {su }}$ is local at a particular prediction of the surrogate model, which depends on the input. The overall distribution of $\varepsilon_{\mathrm{su}}$ is not available over the entire range of the input, thus hindering global sensivity analysis. To overcome this difficulty, an approximate approach is to obtain samples of $\varepsilon_{\mathrm{su}}$ corresponding to samples of random inputs to the surrogate model; these samples of $\varepsilon_{\mathrm{su}}$ are used to construct the overall distribution of $\varepsilon_{\text {su }}$.

\subsubsection{Example: sensitivity analysis on deterministic error and stochastic error}

Suppose that the computational model has the form of

$$
y=f(x)=1.5 x^{2}+6
$$


where $x \sim N(3,1)$ is the input variable. Assume three errors arise in this model:

1. A deterministic error: $\varepsilon_{1}=2 \sin (2 \pi x)$;

2. A stochastic error: $\varepsilon_{2} \sim N(0,1)$;

3. A stochastic error whose distribution depends on $x: \varepsilon_{3} \sim N(0,3-0.4 x)$.

Accounting for all three errors, suppose the corrected model output is

$$
y_{\mathrm{c}}=f(x)+\varepsilon_{1}+\varepsilon_{2}+\varepsilon_{3}
$$

Samples of the corrected model output $y_{\mathrm{c}}$ can be obtained by sampling the input $x$. For a given input, the computational model $f(x)$ is first evaluated. Then the deterministic error $\varepsilon_{1}$ is calculated and the stochastic error $\varepsilon_{2}$ is directly sampled. As for the stochastic error $\varepsilon_{3}$, its standard deviation is first calculated and then $\varepsilon_{3}$ is sampled. Finally, sample values of all three errors are added to $f(x)$ to obtain the corrected model output $y_{\mathrm{c}}$. The PDF of $y_{c}$ is plotted in Figure 7.

\section{Local sensitivity analysis}

The two local sensitivity analysis methods introduced in Section 5.2, which are change of variance and KL-divergence, are used here to assess the contribution of each error to the uncertainty of the corrected model output $y_{\mathrm{c}}$. To obtain $y_{\mathrm{c}}$ without correcting for or without including a particular error, the error is simply fixed at zero in the example. Thus we obtain the conditional $y_{\mathrm{c}}$ with respect to each of the three errors as below: $\left(y_{\mathrm{c}} \mid \varepsilon_{1}=0\right)=f(x)+\varepsilon_{2}+\varepsilon_{3}: y_{\mathrm{c}}$ without correcting for the deterministic error $\varepsilon_{1}$; 
$\left(y_{\mathrm{c}} \mid \varepsilon_{2}=0\right)=f(x)+\varepsilon_{1}+\varepsilon_{3}: y_{\mathrm{c}}$ without including the stochastic error $\varepsilon_{2}$;

$\left(y_{\mathrm{c}} \mid \varepsilon_{3}=0\right)=f(x)+\varepsilon_{1}+\varepsilon_{2}: y_{\mathrm{c}}$ without including the stochastic error $\varepsilon_{3}$.

Similarly, by sampling the input $x$, samples of each conditional $y_{\mathrm{c}}$ are obtained and their PDFs are also plotted in Figure 7. The change of variance is calculated by comparing the sample variance of $y_{\mathrm{c}}$ and that of each conditional $y_{\mathrm{c}}$. And the KLdivergence is calculated by comparing the PDF of $y_{\mathrm{c}}$ and the PDF of each conditional $y_{\mathrm{c}}$. The results are listed in Table 2.

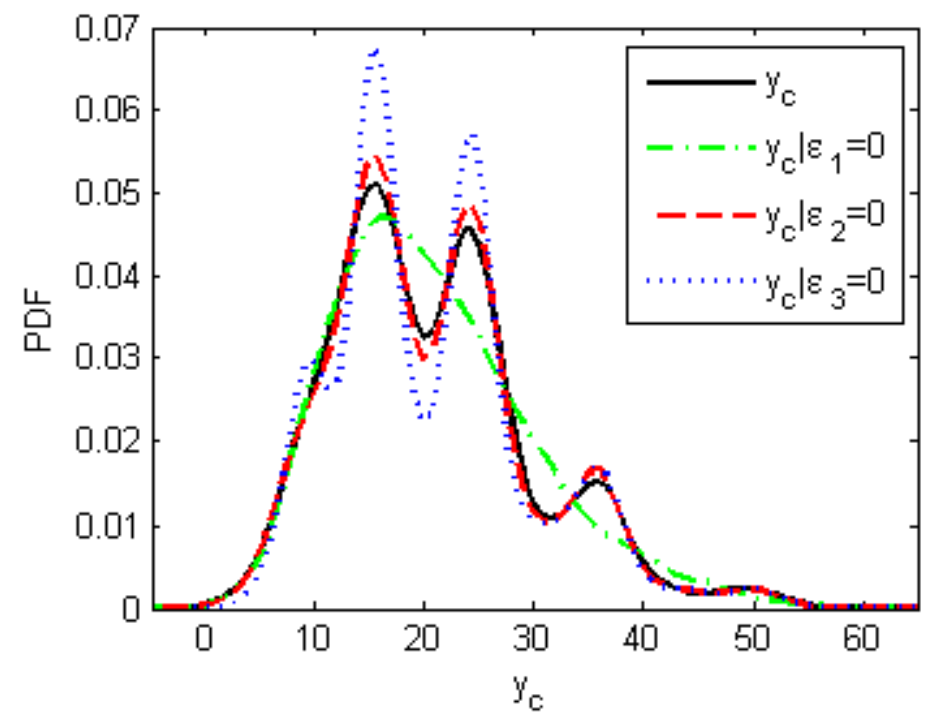

Figure 7. PDFs of $y_{\mathrm{c}}$ and conditional $y_{\mathrm{c}}$

\section{Global sensitivity analysis}

As discussed in Section 5.5, in order to perform global sensitivity analysis, overall distributions must be obtained for the deterministic error $\varepsilon_{1}$ and the stochastic error $\varepsilon_{3}$. Samples of $\varepsilon_{1}$ can be obtained by sampling the input $x$ and the overall distribution of $\varepsilon_{1}$ 
can be estimated from the samples as shown in Figure 8(a). As for $\varepsilon_{3}$ a nested-loop sampling is performed. First the input $x$ is sampled and the standard deviation of $\varepsilon_{3}$ is calculated, and then 10 samples of $\varepsilon_{3}$ are generated. By repeating this procedure 100 times, 1000 samples of $\varepsilon_{3}$ are obtained. Finally the overall distribution of $\varepsilon_{3}$ is estimated from the samples as shown in Figure 8(b). The overall distribution of $\varepsilon_{1}$ and $\varepsilon_{3}$ are both independent of the input $x$.

Based on the above calculation, both $\varepsilon_{1}$ and $\varepsilon_{3}$ are treated as ordinary random variables similar to $\varepsilon_{2}$. This treatment makes calculating the global sensitivity indices possible using Eq. (5.4) and Eq. (5.6), since the fixed value of the error can now be varied according to its distribution. The results are listed in Table 2. In this simple example, all sensitivity analyses give the same ranking of importance among the three errors.
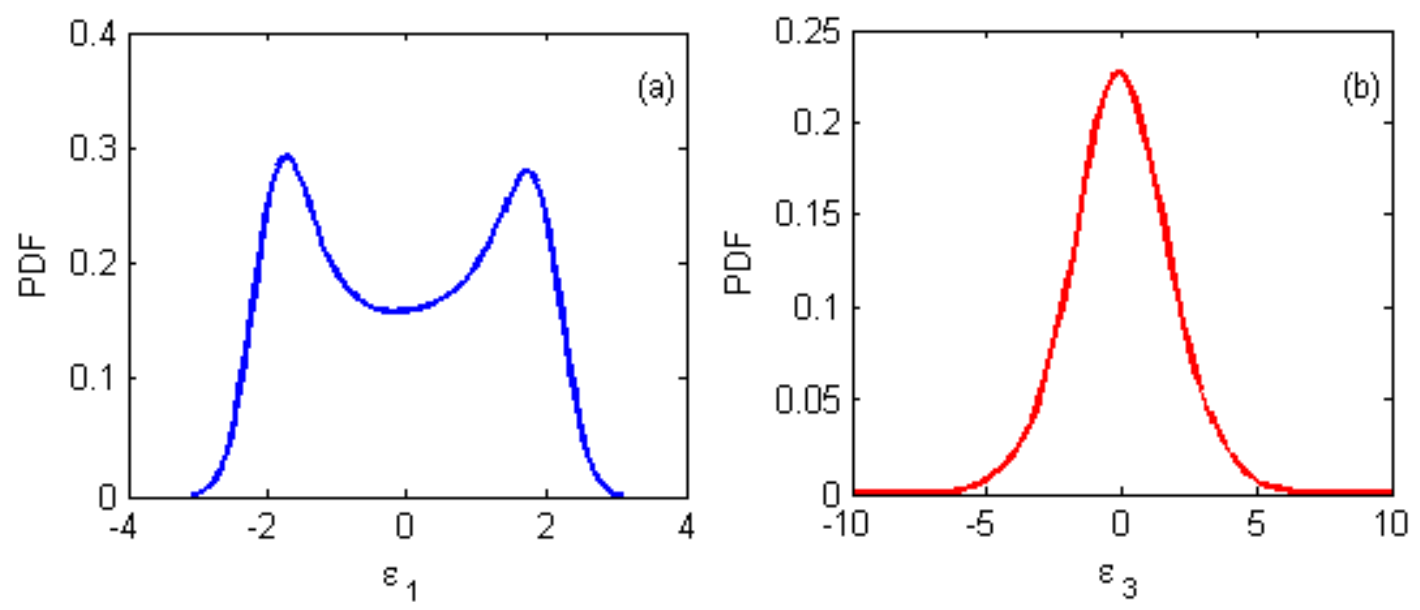

Figure 8. Overall distributions of $\varepsilon_{1}$ and $\varepsilon_{3}$ 
Table 2. Sensitivity analysis results

\begin{tabular}{|c|c|c|c|c|c|c|c|c|}
\hline \multirow{2}{*}{ Error } & \multicolumn{2}{|c|}{$\begin{array}{c}\text { Change of } \\
\text { Variance }\end{array}$} & \multicolumn{2}{c|}{ KL Distance } & \multicolumn{2}{c|}{ Main Effect } & \multicolumn{2}{c|}{ Total Effect } \\
\cline { 2 - 9 } & Index & Ranking & Index & Ranking & Index & Ranking & Index & Ranking \\
\hline$\varepsilon_{1}$ & 1.98 & 2 & 0.55 & 2 & 0.26 & 2 & 0.32 & 2 \\
\hline$\varepsilon_{2}$ & 1.31 & 3 & 0.48 & 3 & 0.15 & 3 & 0.16 & 3 \\
\hline$\varepsilon_{3}$ & 3.40 & 1 & 0.79 & 1 & 0.44 & 1 & 0.51 & 1 \\
\hline
\end{tabular}

\subsection{Summary}

Both local and global sensitivity analysis methods are introduced to identify the contribution of each error to the overall error and thus to rank the important of each error. The ranking is helpful for an efficient model improvement.

The need to compare the various sources of error with the same sensitivity measure creates two issues. Local sensitivity measures are not appropriate for stochastic errors, and calculation of global sensitivity measures is not straightforward for deterministic errors. The proposed treatment in Section 5.5 provides a convenient way of including discretization error and surrogate model error into global sensitivity analysis, thus making the various error sources comparable under the same sensitivity measure. An example is shown in Section 5.6 to demonstrate the proposed procedure. 


\section{CHAPTER VI}

\section{NUMERICAL EXAMPLES}

\subsection{Introduction}

Two numerical examples are used to demonstrate the proposed methods for error quantification. The first example is a cantilever beam model. Quantification of numerical error, model form error and UQ error is demonstrated in this example. The second example considers fatigue crack growth in an airplane wing spar. Error and uncertainty quantification, as well as sensitivity analysis are demonstrated in this example.

\subsection{Cantilever beam}

The tip deflection $\delta$ of a cantilever beam shown in Figure 9 is of interest. The beam has length $L=192 \mathrm{in}$, cross sectional moment of inertia $I=300 \mathrm{in}^{3}$ and Poisson's ratio $v=0.3$. The Young's modulus $E$ is assumed to be a normal random variable with mean value $29000 \mathrm{Ksi}$ and COV 0.06. The self-weight $w(x)$ is assumed to be a onedimensional Gaussian random field, with mean value $75 \mathrm{lb} / \mathrm{in}$ and COV 0.05, as well as an exponential covariance function defined by $C(\Delta x)=\sigma^{2} \exp (-|\Delta x| / b)$, in which $\sigma^{2}$ is the variance and $b$ is the correlation length of the random field. In this example, $b$ is 
assumed to be equal to the length of the beam. The concentrated load $P$ at the beam tip is the input to the model, which is a normal random variable with mean $1000 \mathrm{lb}$ and COV 0.16. For the sake of illustration, assume an error $\varepsilon_{P}$ arises when measuring the input $P$, which is also a normal random variable with zero mean and a variance equal to $20 \%$ of variance of $P$ [31]. Similarly, the output (i.e., deflection) measurement error is also assumed to be a normal random variable with zero mean and standard deviation of 0.01 in .

A finite element model is constructed with 4 beam elements of equal length and a first order polynomial chaos expansion with Hermite bases is constructed as the surrogate computational model.

$$
\delta_{\text {pred }}=h(P, E, w)
$$

9 training points are selected by the collocation method to run the FEA model for training the surrogate model [22]. Then the surrogate model is used to generate samples of model predictions by sampling the inputs. The distribution of model prediction is estimated from the samples to have a mean value of -1.74 and standard deviation of 1.23E-1.

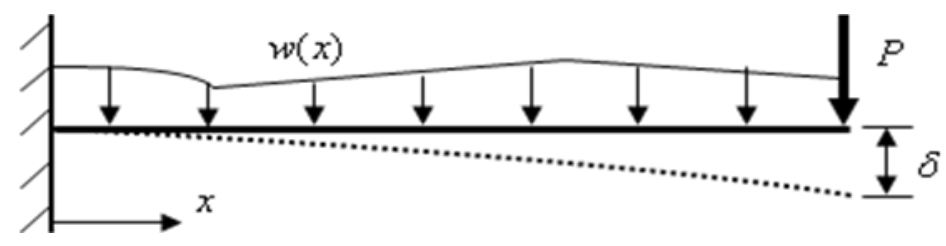

Figure 9. Cantilever beam 


\subsubsection{Numerical error estimation}

In this example, the numerical error in the model prediction is assumed to come from three error sources, surrogate model error $\varepsilon_{\text {su }}$, discretization error in FEA $\varepsilon_{\mathrm{h}}$, and input error $\varepsilon_{P}$. First, a Richardson extrapolation technique is used to correct the discretization error in FEA. For each of the 9 training points, 2 more FEM runs are made with 8 elements and 16 elements, respectively (original number of elements is 4) to calculate $\varepsilon_{\mathrm{h}}$. A new set of 9 training points is obtained by adding the discretization error to the original training points and a new surrogate model $\mathrm{PCE}_{\mathrm{h}}$ is built with the corrected training data. Then $\varepsilon_{P}$ and $\varepsilon_{\mathrm{su}}$ are also included and finally the corrected model prediction $\delta_{\mathrm{c}}$ is given by:

$$
\delta_{\mathrm{c}}=\mathrm{PCE}_{\mathrm{h}}\left(P+\varepsilon_{P}, E, w\right)+\varepsilon_{\mathrm{su}}
$$

The numerical error can be calculated as $\varepsilon_{\text {num }}=\delta_{\mathrm{c}}-\delta_{\text {pred }}$, where $\delta_{\text {pred }}$ is the raw model

prediction. By randomly generating samples of $\varepsilon_{\text {num }}$, the mean and standard deviation of $\varepsilon_{\text {num }}$ are estimated to be $-4.01 \mathrm{E}-3$ and $1.78 \mathrm{E}-1$, respectively.

\subsubsection{Sensitivity analysis of errors}

In this example the computation of change of variance, KL-divergence and main effect index is demonstrated using scatter plots. However calculating the total effect indices cannot be illustrated using scatter plots. 10,000 samples of $\delta_{\text {c }}$ were generated in order to perform the sensitivity analysis. The scatter plots of $\delta_{\mathrm{c}}$ versus $\varepsilon_{P}, \varepsilon_{\mathrm{h}}$ and $\varepsilon_{\mathrm{su}}$ are shown in Figure 10. A vertical thin strip centered at 0 on the horizontal axis is cut 
from each of the scatter plots to calculate the sensitivity indices by the change of variance method and KL divergence method. The widths of the strips are 4, 0.01e-3 and 0.02e-3 for $\varepsilon_{P}, \varepsilon_{\mathrm{h}}$ and $\varepsilon_{\mathrm{su}}$, and would remain the same in the following analysis. 226, 224 and 288 samples of $\varepsilon_{P}, \varepsilon_{\mathrm{h}}$ and $\varepsilon_{\mathrm{su}}$ fall into the strips, respectively. To obtain the change of variance, the variance of $\delta_{\mathrm{c}}$ is calculated from the samples in the strips and is compared against the variance calculated using the total 10,000 samples of $\delta_{\text {c }}$. The results are listed in Table 3. Similarly, KL distances are calculated for each of the three errors by comparing the distributions of the samples in the strips and the overall distribution of $\delta_{\mathrm{c}}$. Then results are listed in Table 3. 

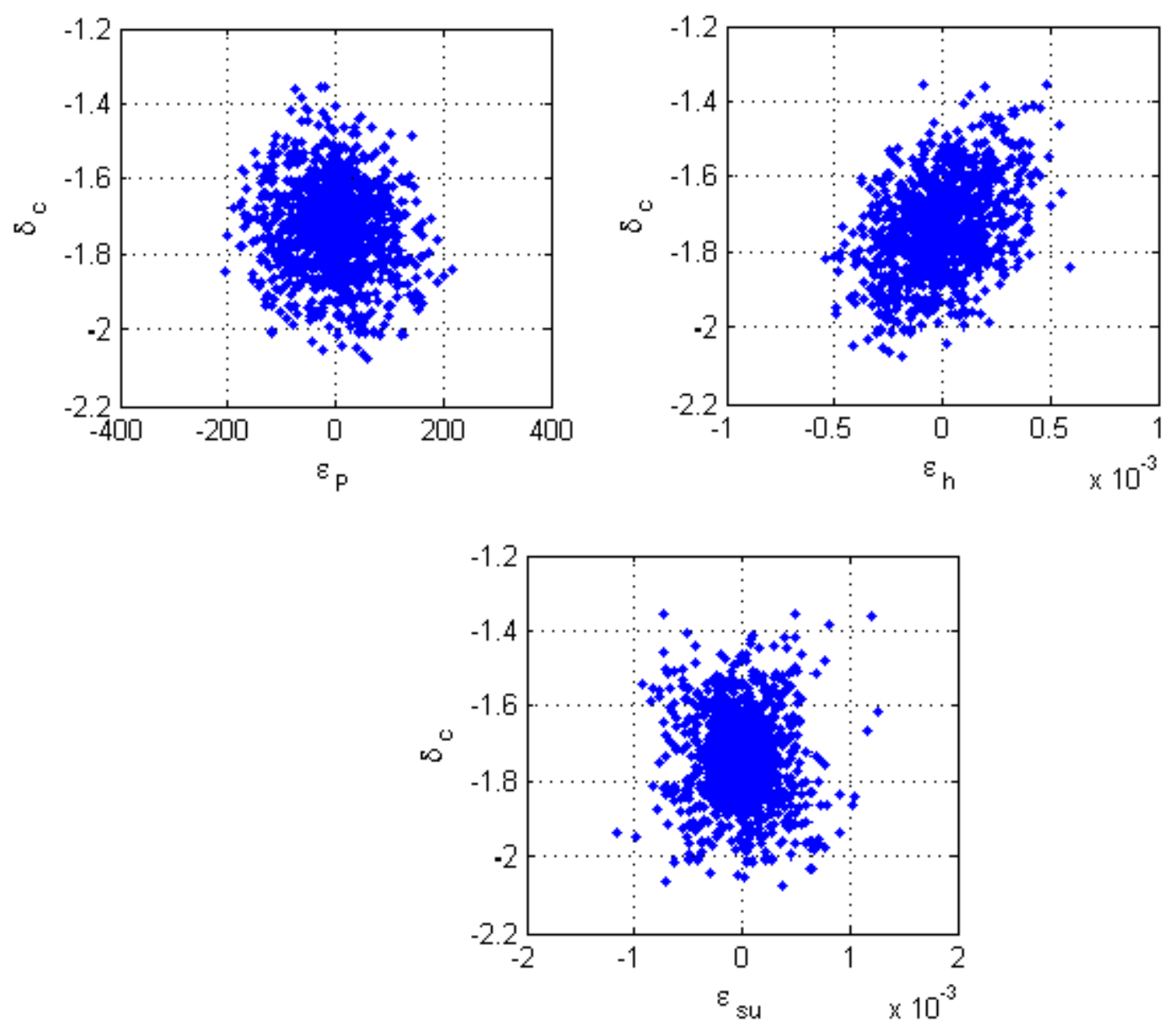

Figure 10. Scatter plots of $\delta_{\mathrm{c}}$ versus $\varepsilon_{P}, \varepsilon_{\mathrm{h}}$ and $\varepsilon_{\mathrm{su}}$.

To calculate the main effect sensitivity index the location of the strip is varied and expected value of $\delta_{\mathrm{c}}$ is calculated within each strip. The scatter plots of the expected values versus the corresponding errors are shown in Figure 11. The main effect sensitivity index is obtained by taking the variance of the expected values. The strip location is varied according to the distribution of each error so as that the variance is calculated with respect to the error. The results are listed in Table 3. For a given input to the model, there 
is only one fixed discretization error. However, as the input to the model varies, the resulting discretization error also varies. Thus the scatter of the discretization error is due to the randomness in the input variables.
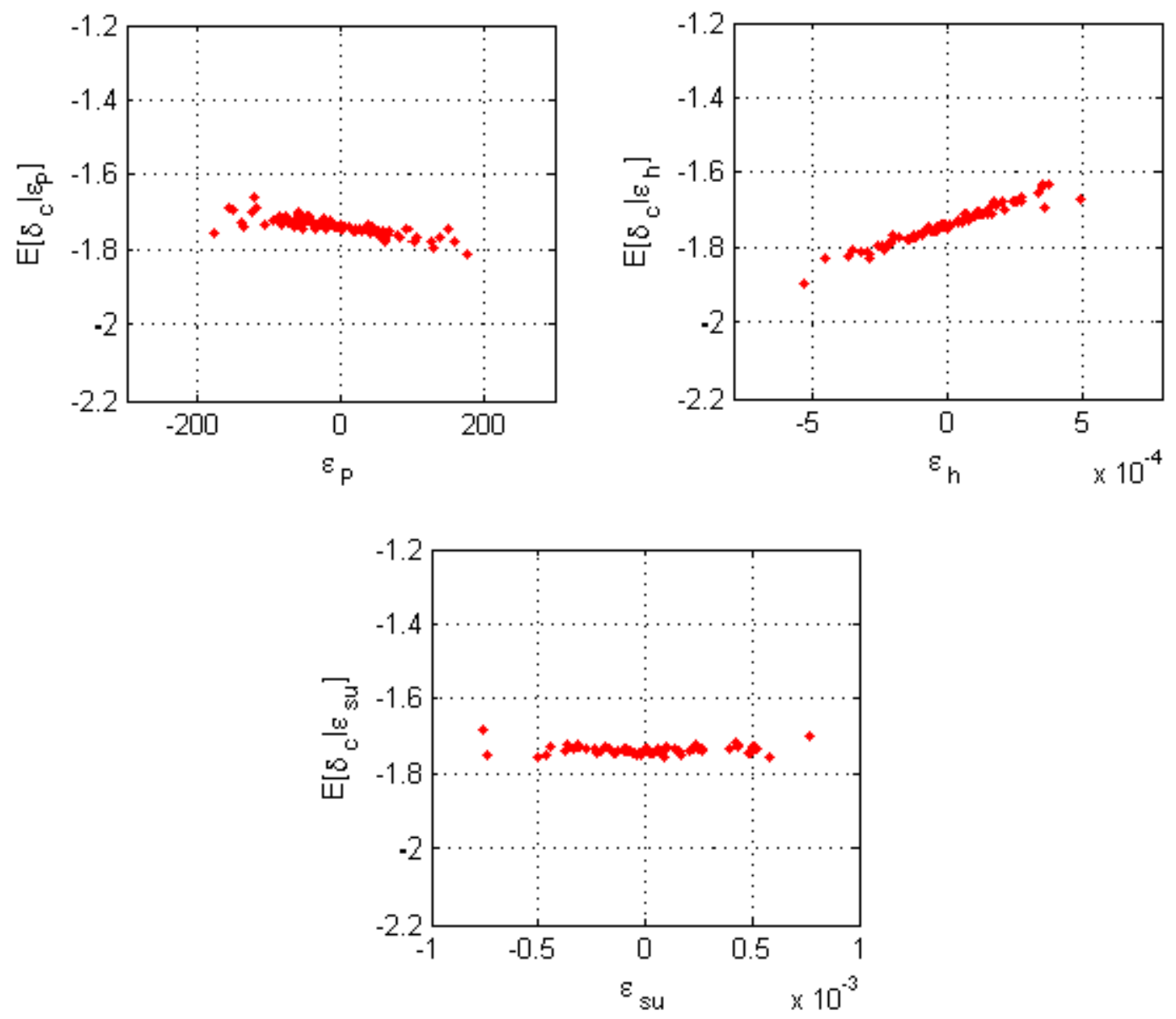

Figure 11. Scatter plots of $E\left[\delta_{\mathrm{c}} \mid \varepsilon\right]$ versus $\varepsilon_{P}, \varepsilon_{\mathrm{h}}$ and $\varepsilon_{\mathrm{su}}$.

Finally the total effect sensitivity indices are computed by Monte Carlo sampling.

The results are also listed in Table 3. It is seen that all methods indicate that the 
discretization error affects the model solution the most.

Table 3. Sensitivity analysis results

\begin{tabular}{|c|c|c|c|c|c|c|c|c|}
\hline \multirow{2}{*}{ Error } & \multicolumn{2}{|c|}{$\begin{array}{c}\text { Change of } \\
\text { Variance }\end{array}$} & \multicolumn{2}{c|}{ KL Distance } & \multicolumn{2}{c|}{ Main Effect } & \multicolumn{2}{c|}{ Total Effect } \\
\cline { 2 - 9 } & Index & Ranking & Index & Ranking & Index & Ranking & Index & Ranking \\
\hline$\varepsilon_{P}$ & $5.35 \mathrm{e}-3$ & 1 & 10.44 & 1 & 0.0296 & 2 & 0.104 & 3 \\
\hline$\varepsilon_{\mathrm{h}}$ & $2.11 \mathrm{e}-5$ & 2 & 5.48 & 2 & 0.1614 & 1 & 0.131 & 1 \\
\hline$\varepsilon_{\mathrm{su}}$ & $1.60 \mathrm{e}-6$ & 3 & 3.50 & 3 & 0.0115 & 3 & 0.126 & 2 \\
\hline
\end{tabular}

\subsubsection{Model form error and UQ error estimation}

Model form error can be estimated from Eq. (4.2); this also needs to take the uncertainty quantification errors into account. Assume 9 experimental observations of beam deflection $\delta_{\text {obs }}$ are available, and assume 20 samples of $\delta_{\mathrm{c}}$ are taken from the error quantification procedure for resampling $\delta_{\mathrm{c}}$. Further assume that the output measurement error $\varepsilon_{\text {om }}$ follows a normal distribution with zero mean and standard deviation 0.01. For comparison, samples of model form error without considering the uncertainty quantification error are also generated by ignoring the sampling errors in Eq. (4.2). The CDFs of model form error with and without considering uncertainty quantification error are plotted in Figure 12 (a). 1000 samples of model form error were generated for each case. The PDFs of sampling error for $\delta_{\mathrm{obs}}$ and $\delta_{\mathrm{c}}$ are also plotted in Figure 12 (b). Statistical results are summarized in Table 4. Since less data is available for $\delta_{\text {obs }}$ than $\delta_{\mathrm{c}}$, the variance of sampling error for $\delta_{\text {obs }}$ is significantly greater than $\delta_{\mathrm{c}}$. It is noticed that 
including the UQ error (due to limited sampling) resulted in an increase in the variance of the estimated model form error since more randomness was introduced. Finally, since the mean and variance of $\varepsilon_{\text {model }}$ with $\varepsilon_{\text {uq }}$ are estimated from 1000 samples, errors arise in their estimates. By the method proposed in Section 3.4, the mean and variance of the mean of $\varepsilon_{\text {model }}$ (including the effect of $\varepsilon_{\text {uq }}$ ) are calculated to be 2.72e-3 and 3.18e-7, respectively; and the mean and variance of the variance of $\varepsilon_{\text {model }}$ (including the effect of $\varepsilon_{\mathrm{uq}}$ ) are calculated to be $5.75 \mathrm{e}-2$ and $1.48 \mathrm{e}-7$, respectively.
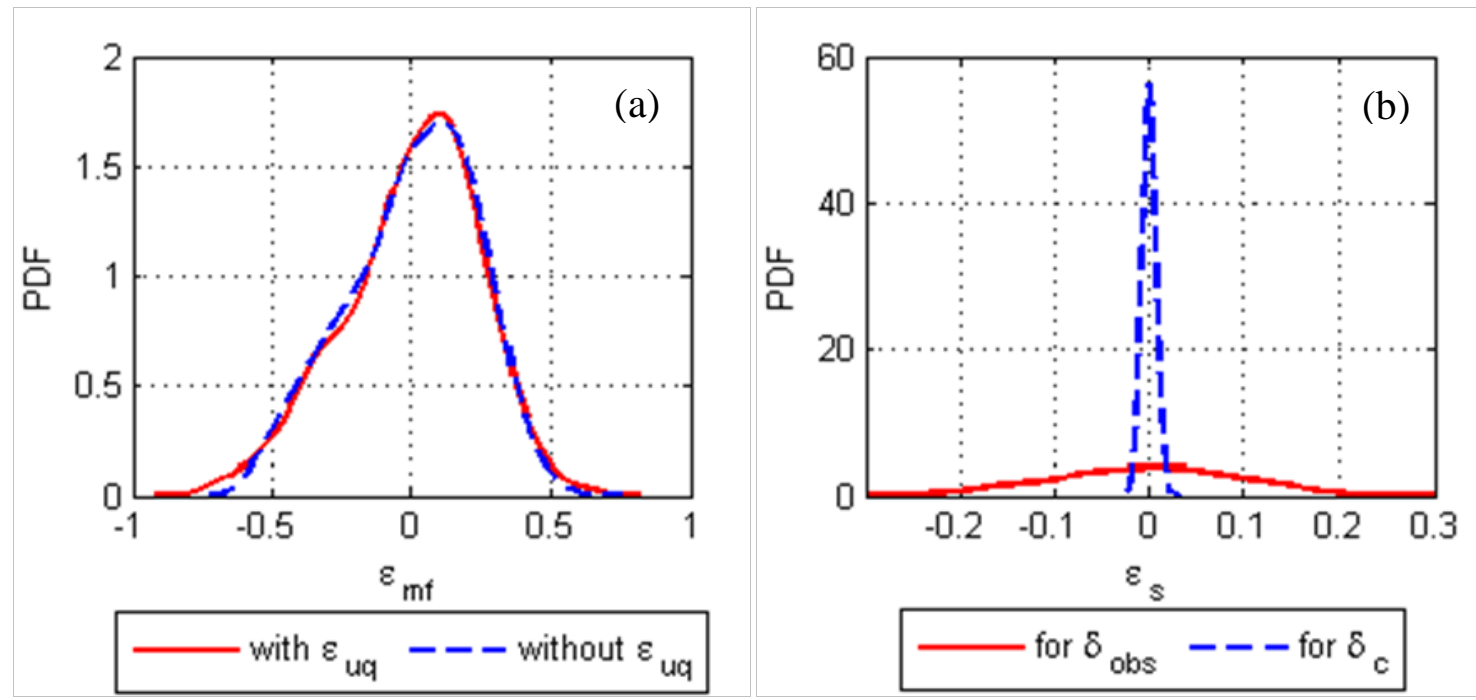

Figure 12. (a): PDFs for model form error (b): PDFs for sampling errors

Table 4. Statistics of model form error and sampling errors

\begin{tabular}{|c|c|c|}
\hline Variable & Mean & Variance \\
\hline$\varepsilon_{\text {model }}$ without $\varepsilon_{\text {uq }}$ & $3.44 \mathrm{e}-3$ & $5.19 \mathrm{e}-2$ \\
\hline$\varepsilon_{\text {model }}$ with $\varepsilon_{\text {uq }}$ & $2.74 \mathrm{e}-3$ & $5.76 \mathrm{e}-2$ \\
\hline$\varepsilon_{\mathrm{s}}$ for $\delta_{\text {obs }}$ & $-7.21 \mathrm{e}-4$ & $1.09 \mathrm{e}-2$ \\
\hline$\varepsilon_{\mathrm{s}}$ for $\delta_{\mathrm{c}}$ & $-0.20 \mathrm{e}-4$ & $5.52 \mathrm{e}-5$ \\
\hline
\end{tabular}




\subsection{Crack growth in an airplane wing spar}

In this example, quantification and sensitivity analysis of errors in crack growth prediction in a part of an airplane wing is of interest. In crack growth analysis, sensitivity analysis of errors faces a major difficulty because different sample values of the same error are input into the analysis in each cycle. This issue will be discussed in detail later. The wing spar plays a key role in connecting the wing to the fuselage, and is subjected to cyclic loading during flight. The final crack size $a_{\mathrm{N}}$ that grows from an initial size $a_{0}=0.05$ in after a given load history is studied. Due to various uncertainties and errors arising in the analysis, $a_{\mathrm{N}}$ is a random variable.

The analysis consists of two modules: structural stress analysis and fatigue crack growth analysis, as shown in Figure 13. The errors and uncertainty considered in this example are input error, discretization error in FEA, surrogate model error, and uncertainty in the crack growth law parameter.

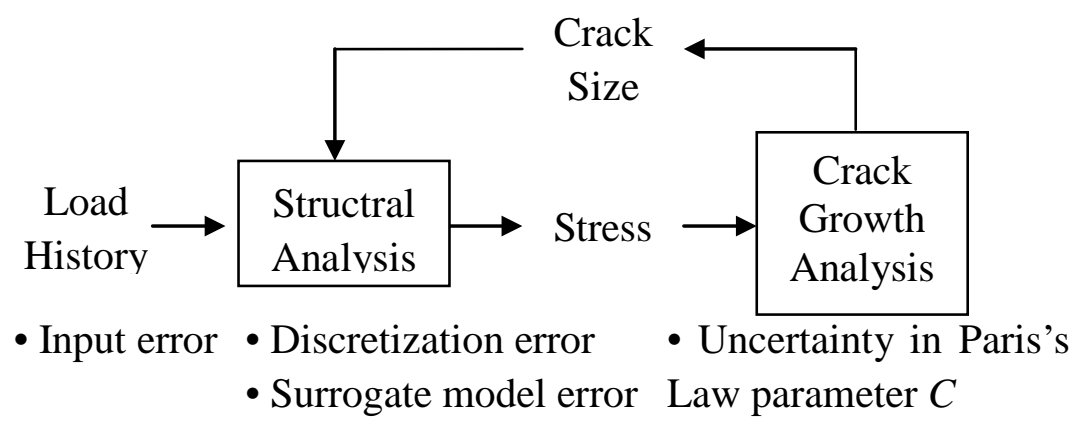

Figure 13. Component analyses and associated sources of error and uncertainty 


\subsubsection{Structural analysis}

In this example, the load history is measured in aerodynamics analysis. The loads on the structure are a bending load $B$ and a pulling load $P$ both over the range of approximately 0 to $400 \mathrm{lb}$. 85,000 load cycles for both loads are recorded as plotted in

Figure 14. During the measuring process, instrumentation errors $\varepsilon_{B}$ and $\varepsilon_{P}$ arise on $B$ and $P$, respectively. This kind of error is modeled as a random error with zero mean, and follows a normal distribution. The standard deviation of $\varepsilon_{B}$ is assumed equal to $30 \%$ of the value of $B$, and the standard deviation of $\varepsilon_{P}$ is equal to $15 \%$ of the value of $P$.

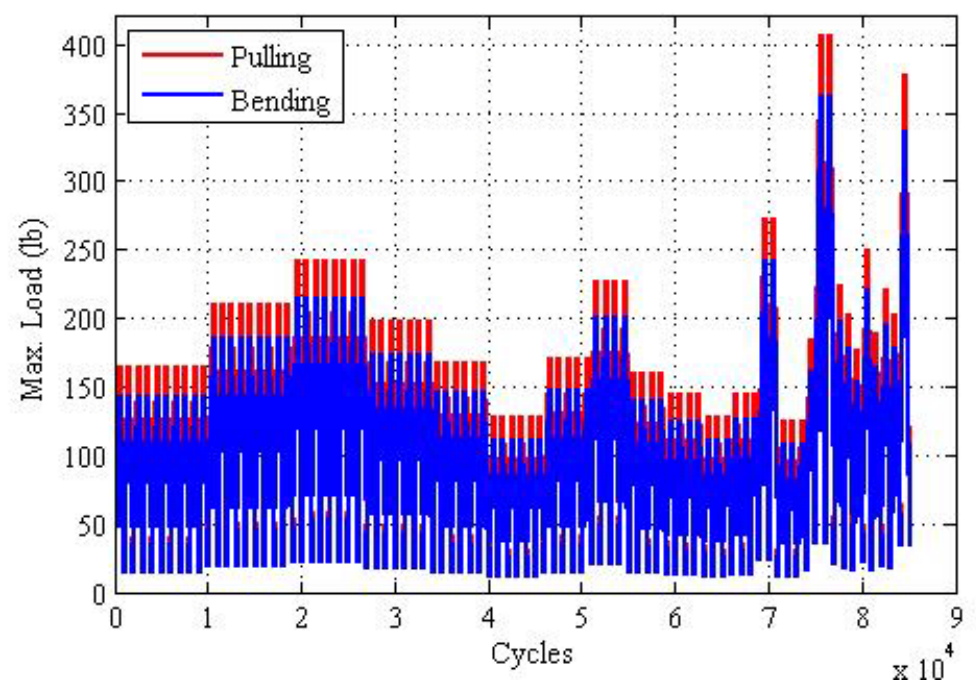

Figure 14. Loading history on the structure

The loads $B$ and $P$ are inputs to the structural model. The output of structural analysis is the first principal stress in the critical region (hot spot). The analysis is carried 
out by a surrogate model trained by 9 FEA runs. To quantify the discretization errors in the finite element analysis using Richardson extrapolation, for each of the training points the finite element analysis is repeated with finer meshes with two levels of refinement. The refinement ratio is 0.75 , which means the element size is multiplied by 0.75 in each refinement.

\subsubsection{Crack growth analysis}

Before proceeding with the crack growth analysis, the stress intensity factor $\Delta K$ is calculated first from the structural analysis result (in the context of linear elastic fracture mechanics). This is given by

$$
\Delta K=\beta \Delta s \sqrt{\pi a}
$$

where $s$ is the stress at crack tip, $a$ is the crack length, and $\beta$ depends geometry and crack length. In this example, $\beta$ available from [32]. The Paris' law is used here to illustrate the crack growth calculation:

$$
\frac{d a}{d N}=C(\Delta K)^{n}
$$

The parameter $n$ is assumed to be deterministic at 3.2. Due to fitting error the parameter $C$ is assumed to be a random variable that follows a lognormal distribution, with a mean 1.0E-7 and a COV of 0.24 . 


\subsubsection{Correction of errors}

The following states the procedure of calculating $a_{N}$ that is corrected for all error. At the beginning, a random sample of $C$ is generated, which stays constant throughout all cycles. In each cycle, first the mean values of $B$ and $P$ are obtained from a given variable amplitude load history. Random samples of $\varepsilon_{B}$ and $\varepsilon_{P}$ are generated, and are added to $B$ and $P$, respectively. The corrected $B$ and $P$ are then fed into the surrogate model. The surrogate model outputs the stress prediction $s$, as well as the associated prediction error. Next Eq. (6.5) is used to calculate the stress intensity factor, which is input to the crack growth law. To accelerate the computation, the increment of crack length $d a$ is calculated in blocks of every 10 cycles, i.e., $d N$ is set to 10 in Eq. (6.6). Finally, the crack length is updated until the end of the load history. The analysis is repeated to generate 10,000 such samples of $a_{N}$.

\subsubsection{Results and sensitivity analysis}

The $a_{N}$ obtained in the previous section that is corrected for all errors is denoted as $a_{c}$ in the following discussion. However, the structure of the crack growth problem is not like that of the previous example due to the cycle by cycle analysis. A small example would explain this special case. Let $a_{i+1}=g\left(a_{i}\right)+\varepsilon$ be a crack growth model, in which $a_{i}$ and $a_{i+1}$ are the crack sizes in the previous and the next cycle, and $\varepsilon$ is a random error. Supposed that after three cycles the crack grows from $a_{0}$ to $a_{3}$, we have 


$$
a_{3}=g\left(g\left(g\left(a_{0}\right)+\varepsilon^{(1)}\right)+\varepsilon^{(2)}\right)+\varepsilon^{(3)}
$$

in which $\varepsilon^{(1)}, \varepsilon^{(2)}$ and $\varepsilon^{(3)}$ are three samples of the error $\varepsilon$ but they are generated independently. Or we can say $a_{3}$ is function of four input variables $a_{3}=g_{3}\left(a_{0}, \varepsilon^{(1)}, \varepsilon^{(2)}, \varepsilon^{(3)}\right)$. The existing sensitivity methods are only able to estimate the sensitivity of $a_{3}$ with respect to each of $\varepsilon^{(1)}, \varepsilon^{(2)}$ and $\varepsilon^{(3)}$ individually. However, the sensitivity of $a_{3}$ to the overall error $\varepsilon$ is what we are actually interested in. An approximate approach to overcome this hurdle is to assume that all samples of the same error have the same value throughout all cycles. This makes it possible to compute the sensitivity with respect to a single error term $\varepsilon$ over all cycles.

The results in Table 5 show that all four sensitivity measures are able to indicate that the final crack size is most sensitive to the parameter $C$ in Paris's Law, and is least sensitive to the discretization error in FEA. This implies that in order to achieve a more accurate prediction of the final crack size, a more precise parameter $C$ (i.e., narrower scatter) is needed. In contrast, the discretization error in FEA is almost negligible.

Table 5. Sensitivity analysis results

\begin{tabular}{|c|c|c|c|c|c|c|c|c|}
\hline \multirow{2}{*}{$\begin{array}{c}\text { Error } \\
\text { /Uncertianty }\end{array}$} & \multicolumn{2}{|c|}{$\begin{array}{c}\text { Change in } \\
\text { variance }\end{array}$} & \multicolumn{2}{c|}{ K-L divergence } & \multicolumn{2}{c|}{ Main Effect } & \multicolumn{2}{c|}{ Total Effect } \\
\cline { 2 - 9 } & Index & Ranking & Index & Ranking & Index & Ranking & Index & Ranking \\
\hline$\varepsilon_{B}$ & $0.17 \mathrm{e}-3$ & 4 & 6.85 & 3 & $4.69 \mathrm{e}-2$ & 4 & $8.36 \mathrm{e}-1$ & 4 \\
\hline$\varepsilon_{P}$ & $0.32 \mathrm{e}-3$ & 3 & 6.29 & 4 & $4.18 \mathrm{e}-1$ & 2 & $9.13 \mathrm{e}-1$ & 2 \\
\hline$C$ & $2.95 \mathrm{e}-3$ & 1 & 37.54 & 1 & $9.69 \mathrm{e}-1$ & 1 & $9.88 \mathrm{e}-1$ & 1 \\
\hline$\varepsilon_{\mathrm{su}}$ & $2.82 \mathrm{e}-3$ & 2 & 36.63 & 2 & $3.80 \mathrm{e}-1$ & 3 & $9.09 \mathrm{e}-1$ & 3 \\
\hline$\varepsilon_{\mathrm{h}}$ & $8.36 \mathrm{e}-5$ & 5 & 6.15 & 5 & $1.07 \mathrm{e}-3$ & 5 & $2.48 \mathrm{e}-1$ & 5 \\
\hline
\end{tabular}




\section{CHAPTER VII}

\section{CONCLUSION}

This research studied some of the errors that arise in mechanics computational models. There are three major contributions in this research. The first is that the errors are clearly separated and a quantification method is developed for each of the errors, including model form error and three typical sources of numerical error. Some of these errors are random and some are deterministic. Deterministic error is corrected by adding it to the prediction; and random error is included in the model output through sampling. By correcting or accounting for all the errors the corrected model output is obtained. The corrected model outputs together with observed data are used to estimate model form error through sampling, where uncertainty quantification error arises. A methodology to quantify and propagate the UQ error is developed (this is the second contribution). Moreover, based on the quantification of UQ error an efficient method is proposed for interval estimation for mean and variance of given limited number of samples.

The third major contribution of this research is that sensitivity analysis methods are developed to rank the contribution of each error. Past work in global sensitivity analysis has only considered the influence of input random variables on output 
uncertainty; this research extends the methods to include model errors. Two local and two global probabilistic sensitivity measures (three variance-based and one entropy-based) are adopted. Approximation approaches are developed for fatigue crack growth analysis that requires repetition of error terms in each cycle. The proposed error quantification and sensitivity analysis framework would be beneficial for multi-level models to help determine where low fidelity analyses are sufficient and where high fidelity analyses are needed, thus balancing computational effort and prediction accuracy.

In this research multi-disciplinary models with feed-back coupling relationships are not considered. However, such models are commonly seen, such as in fluid-structure interaction problems, where the output of one model is the input to the other model, and several iterations are needed until the solution converges. Errors arise in both models, and accumulate through the iterations between the two models. An efficient and accurate methodology to quantify the solution error in this case needs to be developed.

Other sources of error need to be considered in this methodology, such as truncation and round-off errors. Also, some of the errors considered, such as experimental errors and solution errors can be decomposed further into several components, related to different experiments or different computational modules. Future work needs to address this, especially for multi-level, multi-scale, multi-physics models. However, the methodology developed in this thesis can still serve as a template for combining the various sources of error and uncertainty. 


\section{REFERENCES}

1. Rebba, R. Model validation and design under uncertainty. Nashville, TN: Vanderbilt University, 2007.

2. Oberkamf, W. L., and Trucano, T. G. Verification and validation in computational fluid dynamics. Progress in Aerospace Sciences, Vol. 38 (3), pp. 209-272, 2002.

3. American Institute of Aeronautics and Astronautics. Guide for the verification and validation of computational fluid dynamics simulations. AIAA-G-077-1998, Reston, VA, 1998.

4. Oden, J. T., Babuska, I., Nobile, F., Feng, Y., and Tempone, R., Theory and methodology for estimation and control of errors due to modeling, approximation, and uncertainty. Comput. Methods Appl. Mech. Engrg, 194(2005) 195-204.

5. Rebba, R., Mahadevan, S., and Huang, S. P. Validation and error estimation of computational models. Reliability Engineering and System Safety. Vol. 91, pp. 13901397, 2006.

6. Alvin, K. F. A method for estimating discretization error in non-deterministic analysis. AIAA Journal, Vol. 38 (5), pp. 910-916, 2000.

7. Babuska, I., and Chatzipantelidis, P., “On solving elliptic stochastic partial differential equations. Comput. Methods Appl. Mech. Engg. Vol. 19 (37-38), pp. 40934122, 2002.

8. Ayyub, B. M., and Haldar, A. Decisions in construction operation. Journal of the Construction Engineering and Management Division, ASCE, Vol. 111 (4), pp. 343-357, 1985.

9. Shooman, M. L. Probabilistric reliability: an engineering approach. McGraw-Hill, New York, 1968.

10. Efron, B. and Tibshirani, R. Bootstrap methods for standard errors, confidence intervals, and other measures of statistical accuracy. Statistical Science, Vol. 1 (1), pp. 5475, 1986. 
11. DeBrota, D. J., Swain, J. J., Roberts, S. D., and Venkataraman, S. input modeling with the Johnson System of distributions. In Proceedings of the 1988 Winter Simulation Conference. 1988.

12. Babuska, I., and Rheinboldt, W. C. A posterori error estimates for the finite element method. Int. J. Numer. Methods Engrg. Vol. 12, pp. 1597-1615, 1978.

13. Demkowicz, L., Oden, J. T., and Strouboulis, T. Adaptive finite elements for flow problems with moving boundaries. Part 1: Variational principles and a posteriori error estimates, Comput. Methods Appl. Mech. Engrg. Vol. 46, pp.201-251.

14. Zienkiewicz, O.C., and Zhu, J.Z. A simple error estimator and adaptive procedure for practical engineering analysis, Int. J. Numer. Methods Engrg. Vol. 24, pp. 337-357, 1987.

15. Szabo, B.A. Mesh design for the p version of the finite element, Comput. Methods Appl. Mech. Engrg. Vol. 55, pp. 181-197, 1989.

16. Ainsworth, M., and Oden, J. T., A posteriori error estimation in finite element analysis, Comput Methods Appl Mech Eng 142 (1997), pp. 1-88.

17. Richardson, L. F., 1910, "The Approximate Arithmetic Solution by Finite Differences of Physical Problems Involving Differential Equations, with Application to Stresses in a Masonry Dam," Transactions of the Royal Society of London, Series A., Vol. 210, pp. 307-357

18. Roache, P. J. Verification and validation in computational science and engineering. Hermosa publishers, Albuquerque, NM, 2002.

19. Richards, S. A. Completed Richardson extrapolation in space and time. Communications in Numerical Methods in Engineering, Vol. 13, pp. 573-582, 1997.

20. Celik, I., and Karatekin, O., Numerical experiments on application of Richardson Extrapolation with nonuniform grids. Journal of Fluids Engineering, Vol. 119, pp. 584590, 1997.

21. Isukapalli, S. S. Uncertainty Analysis of Transport-Transformation. Ph.D. thesis, Rutgers, the State University of New Jersey, 1999.

22. Huang, S. P., Mahadevan, S., and Rebba, R. Collocation-based stochastic finite element analysis for random field problems. Probabilistic Engineering Mechanics, Vol. 
22, Issue 2, pp. 194-205, 2007

23. Seber, G. A. F, and Wild, C. J. Nonlinear Regression. John Wiley \& Sons, Inc., 1989.

24. Rasmussen, C. E., and Williams, C. K. I. Gaussian Processes for Maching Learning. The MIT Press, 2006.

25. Saltelli, A., Chan, K., and Scott, E. M. Sensitivity Analysis, John Wiley \& Sons, Chichester, New York, 2000.

26. Sobol', I. M., Global sensitivity Indices for nonlinear mathematical models and their Monte Carlo estimates. Mathematics and Computers in Simulation. Vol. 55, pp. 271280, 2001.

27. Liu, H., Chen, W., and Sudjianto, A. Relative entropy based method for probabilistic sensitivity analysis in engineering design. J. Mechanical Design. Vol. 128, pp. 326-336, 2006.

28. Felli, J. C. and Hazen, G. B. Sensitivity analysis and the expected value of perfect information. Medical Decision Making, Vol. 18, pp. 95-109, 1998 (Erratum in Vol. 23 (1) pp. 97, 2003).

29. Krzykacz-Hausmann, B. Epistemic sensitivity analysis based on the concept of entropy. In Proceedings of SAMO 2001, Edited by P. Prado and R. Bolado, Madrid, CIEMAT, pp. 31-35, 2001.

30. Kullback, S. and Leibler, R.A. On information and sufficiency. The Annals of Mathematical Statistics. Vol. 22 (1), pp. 79-86, 1951.

31. Aeschliman, D. P., Oberkampfi, W. L. Experimental Methodology for Computational Fluid Dynamics Code Validation. Sandia Report SAND95-I 189 UC-706, 2006.

32. Anderson, T. L. Fracture mechanics: fundamentals and applications (3rd Ed.). Taylor and Francis, 2005.

33. Ghanem, R. G. and Spanos, P. Stochastic finite elemnets: a spectral approach. Springer, Berlin, 1991.

34. Haldar, A. and Mahadevan, S. Probability, reliability and statistical methods in 
engineering design. John Wiley and Sons, Inc., New York, 2000.

35. Bonett, D. G., Approximate confidence interval for standard deviation of nonnormal distributions, Computational Statistics \& Data Analysis. Vol. 50, pp. 775 782, 2006.

36. Cojbasic, V., Tomovic, A. Nonparametric confidence intervals for population variance of one sample and the difference of variances of two samples, Computational Statistics \& Data Analysis. Vol. 51, pp. 5562 - 5578, 2007.

37. Saltelli, A., Ratto, M. and Andres, T. et. al. Global sensitivity analysis: the primer. John Wiley and Sons, Ltd., England. 2008.

38. Saltelli, A. Making best use of model valuations to compute sensitivity indices. Computer Physics Communications. Vol. 145, pp. 280-297. 2002.

39. Belendez, T., Neipp, C., and Belendez, A. Large and small deflections of a cantilever beam, European Journal of Physics, Vol. 23, pp. 371-379, 2002. 